\title{
Spherical subcomplexes of spherical buildings
}

\author{
BERND SCHULZ
}

Let $\Delta$ be a thick, spherical building equipped with its natural CAT(1) metric and let $M$ be a proper, convex subset of $\Delta$. If $M$ is open or if $M$ is a closed ball of radius $\pi / 2$, then $\Lambda$, the maximal subcomplex supported by $\Delta \backslash M$, is $\operatorname{dim} \Lambda$-spherical and non-contractible.

\section{E24; $11 \mathrm{~F} 75$}

Connectivity properties of subcomplexes in spherical buildings play an important role in establishing finiteness properties of $S$-arithmetic groups. The complexes arise as relative links of filtrations of Euclidean buildings. The main result of this paper is the sphericity of open and closed hemisphere complexes in spherical buildings.

Let $\mathrm{M}$ be a subset with property $(\mathrm{P})$ of a geometrically realized spherical building. The maximal subcomplex contained in $\mathrm{M}$ is called a $(\mathrm{P})$ supported subcomplex. Recall that a simplicial complex $\Lambda$ is homotopy Cohen-Macaulay, if the link of every simplex $\sigma$ of $\Lambda$ (including the empty simplex) is $(\operatorname{dim} \Lambda-\operatorname{dim} \sigma-1)-$ spherical. The present paper uses the theory of abstract spherical buildings and their metric realizations in order to proof the following two theorems.

Theorem A Non-empty, closed, coconvex supported subcomplexes of spherical buildings are homotopy Cohen-Macaulay. They are non-contractible for at least onedimensional, thick buildings.

The subcomplex supported by the complement of a closed (resp. an open) ball with radius $\pi / 2$ is called an open (resp. a closed) hemisphere complex. Note, that closed hemisphere complexes are closed, coconvex supported subcomplexes. Their sphericity was also independently proved by J Dymara and D Osajda [18].

Theorem B Open hemisphere complexes of thick spherical buildings are homotopy Cohen-Macaulay and non-contractible.

In the late 1980's, P Abramenko [3] and H Abels [1] independently determined the finiteness length of $\operatorname{SL}_{n}\left(\mathbb{F}_{q}[t]\right)$ provided that $q$ is sufficiently big compared with $n$. Later on, Abramenko [4] generalized the result to absolutely almost simple classical 
$\mathbb{F}_{q}$-groups of positive rank over $\mathbb{F}_{q}[t]$. The proofs used the action of these groups $\Gamma$ on a simplicial Euclidean building $X$ and the existence of a cocompact $\Gamma$-filtration of $X$ with spherical relative links. Once such a filtration was established, the finiteness length of $\Gamma$ followed by Brown's Criterion [11, Corollary 3.3]. Since links in Euclidean buildings are spherical buildings, the search for spherical subcomplexes of spherical buildings was a key problem. Specifically the restrictions on $q$ in the above results have been made to get the desired connectivity properties of the relative links.

The proof of $\mathrm{H}$ Behr's characterization of finitely generated and finitely presented $S$-arithmetic groups over function fields [7] used similar geometric arguments. The situation was more complicated, because in view of the groups that paper treated the general case. So $X$ was not simplicial and one needed reduction theory to define a cocompact $\Gamma$-filtration. Since [7] aimed on finite presentation it was sufficient to show that relative links are simply connected without dependencies on $q$. As in [3] and [4], the verification of the connectivity properties occupied the most part of the article. Anyway, Behr's result and its proof suggested that a generalization of the above results using a suitable filtration of $X$ is possible. The obstacle was that one only knew a sparse collection of spherical complexes that could serve as relative links in that general context.

Besides sphericity, subcomplexes of a spherical building $\Delta$ that could serve as relative links admit the action of a parabolic subgroup $P$ of $\operatorname{Aut}(\Delta)$. In Behr [7] sphericity has been proofed for the most candidates up to $\operatorname{dim} \Delta \leq 2$. The method was to show that $P$ can be generated or even described as an amalgamated sum of the $P$-stabilizers that belong to the vertices of a fundamental domain for the action of $P$ (see $\mathrm{J}$ Tits [27, 8. Corollaire 1]). Any heretofore known complex of higher dimension that could serve as a relative link has been investigated in the second part of Abramenko [4]. Using the flag complex models of classical spherical buildings Abramenko examined the complexes $\Delta^{\mathrm{o}}(\sigma)$ for a simplex $\sigma$ of $\Delta$; that is the subcomplex whose chambers are the chambers of $\Delta$ containing an opposite of $\sigma$. One knew from other contexts, for instance $\mathrm{K}$ Vogtmann [28], that $\Delta^{\mathrm{o}}(\sigma)$ is spherical without dependency on $q$ for some types of buildings and simplices. But it turned out that this is not true in general.

These examples have not been sufficient to indicate how a filtration that works in the general case of $S$-arithmetic groups over function fields could be constructed. The aim of this paper is to provide a wide range of subcomplexes of spherical buildings that could serve as relative links. In order to reduce the complexity of defining a filtration the complexes should have a uniform description like the $\Delta^{\mathrm{o}}(\sigma)$ that occurred as relative links in Abels [1] and Abramenko [4]. Behr [7] demonstrated that the invariants of the Behr-Harder reduction theory give rise to a filtration defined by terms of metric geometry. Hence, the candidates for the relative links should have a metric description. 
The observation that the most of the above examples that are spherical regardless of $q$ can be described as a complex supported by the complement of a closed ball with radius $\pi / 2$ leaded to the investigation of hemisphere complexes.

In [13] the upper bound of the finiteness length of $S$-arithmetic groups over function fields has been determined by K-U Bux and K Wortman without local topological arguments. But in [14] the same authors used Theorems A and B in order to generalize the result of U Stuhler [25] on the finiteness length of $\mathrm{SL}_{2}$ over an $S$-arithmetic ring to absolutely almost simple rank-1-groups. The idea was to construct a cocompact $\Gamma$-complex from $X$ by collapsing disjoint horoballs. To prove that this complex is sufficiently high connected, Bux and Wortman showed that horospheres in $X$ are spherical applying the sphericity of hemisphere complexes.

Two years later K-U Bux, R Köhl (né Gramlich) and S Witzel [15] removed the restrictions to the classical types and on $q$ in the result of Abramenko [4] on the finiteness properties of absolutely almost simple $\mathbb{F}_{q}$-groups over $\mathbb{F}_{q}[t]$. In that paper the authors returned to Abramenko's approach and succeed in constructing a cocompact $\Gamma$-filtration whose relative links are hemisphere complexes or akin to closed, coconvex supported subcomplexes. Shortly afterwards Witzel [29] advanced the result to groups over $\mathbb{F}_{q}\left[t, t^{-1}\right]$ by extending the filtration of [15] to the case of non-simplicial Euclidean buildings. Finally Bux, Köhl and Witzel [16] treated the general case by improving the filtration of [29] using reduction theory and determined the finiteness length of $S$-arithmetic groups over function fields again applying the sphericity of hemisphere complexes.

Acknowledgements The current paper is a shortened version of my thesis [23] written at the Johann Wolfgang Goethe-Universität in Frankfurt am Main. I would like to thank Peter Abramenko, my doctoral advisor Helmut Behr and Kai-Uwe Bux for helpful discussions and their support on writing my thesis. I am indebted to Kai-Uwe Bux for commenting on earlier versions of the present paper and for suggesting valuable improvements.

\section{Notation, conventions and recalls}

\subsection{Simplicial complexes}

We identify simplicial complexes with their geometric realization. The sets of vertices and simplices of a simplicial complex $X$ will be denoted by $\operatorname{vt}(X)$ and $\mathcal{S}(X)$, respectively. Simplices are open (in their closure). St $\sigma$ denotes the star of a simplex $\sigma$. The star of a point is the star of the simplex carrying that point. The link $\operatorname{Lk} \sigma$ of a 
simplex $\sigma \in \mathcal{S}(X)$ is the subcomplex of $X$ whose simplices $\tau$ are disjoint from $\sigma$ but the upper bound $\sigma \cup \tau$ exists. We will write $\operatorname{Lk}_{X} \sigma$ and $\mathrm{St}_{X} \sigma$ if $X$ is a subcomplex. The join of two simplicial complexes $X, Y$ will be denoted by $X * Y$.

\subsection{Construction of spherical simplicial complexes}

We adopt the definitions from Quillen [22, Section 8]. A simplicial complex is $n-$ spherical (or spherical) if it is $n$-dimensional and $(n-1)$-connected. By convention non-empty complexes are $(-1)$-connected. The empty complex is $(-1)$-dimensional and (-2)-connected. A simplicial complex $X$ is said to be homotopy Cohen-Macaulay if $\operatorname{Lk} \sigma$ is $(\operatorname{dim} X-\operatorname{dim} \sigma-1)$-spherical for every simplex $\sigma \in \mathcal{S}(X)$ (including the empty set $\varnothing \in \mathcal{S}(X))$.

A commonly used way to show connectivity properties of simplicial complexes is to build these complexes from complexes with known connectivity properties. An overview of the necessary methods can be found in Björner [9, Sections 9 and 10]. A common method is to build up joins, because the joins of spherical complexes are known to be spherical (see Abramenko [3, Korollar zu Bemerkung 6], see also Vogtmann [28, Proof of 1.1]). The following generalization of the gluing lemma (see Björner [9, Lemma 10.3]) is also a standard tool. It is a consequence of the Hurewicz isomorphy theorem (see Spanier [24, page 398]), the Mayer-Vietoris sequence of reduced homology, and Van Kampen's theorem (see Hilton and Wylie [20, 6.4.3]).

Lemma 1.1 (Gluing Lemma) Let $I$ be an index set. Let $X$ and $Y_{i}$ for $i \in I$ be subcomplexes of a simplicial complex $Z=X \cup \bigcup_{i \in I} Y_{i}$. Assume $Y_{i} \cap Y_{j} \subseteq X$ for all $i, j \in I$ with $i \neq j$.

(a) If $X$ and $Y_{i}$ are $n$-connected and $X \cap Y_{i}$ is (n-1)-connected for all $i \in I$, then $Z$ is $n$-connected.

(b) If $Z$ and $X \cap Y_{i}$ are $n$-connected for all $i \in I$, so is $X$.

To show $n$-connectedness of a connected simplicial complex, it is sufficient to prove that every finite subcomplex is contained in a $n$-connected subcomplex, because continuous images of spheres and balls are contained in finite subcomplexes (by compactness). Since the metric topology and the weak topology coincide on finite subcomplexes, one may use the metric topology.

\subsection{Spherical buildings}

Geometrically realized spherical buildings $\Delta$ admit a unique metric, invariant under automorphisms, such that apartments are isometric to the $\operatorname{dim} \Delta$-dimensional unit 
sphere by Bridson and Haefliger [10, II.10 Theorem 10A.4]. We will denote the corresponding canonical metric by $d$ (or by $d_{\Delta}$ if it is necessary to avoid confusions). $(\Delta, d)$ is complete and CAT(1). Isomorphisms of apartments induce isometries and $d$ is a length metric.

In most considerations we do not need to consider the cardinality of chambers containing a given panel. But some crucial constructions need a thick building. Note that the main results do not hold for weak buildings. Thus, for simplicity, we agree that buildings are thick throughout this paper.

According to Abramenko and Brown [5, Proposition 12.18], retractions onto apartments are distance decreasing. We record the precise statement.

Proposition 1.2 The retraction $\rho=\rho_{\Sigma, C}: \Delta \rightarrow \Sigma$ onto $\Sigma$ centered at $C$ is distance decreasing for every apartment $\Sigma$ and every chamber $C$ of $\Sigma$, that is, $d(\rho(x), \rho(y)) \leq$ $d(x, y)$ for all $x, y \in \Delta$. Equality holds if $x \in \bar{C}$.

Notation 1.3 For $x, y \in \Delta$ we put $[x, y]=\{z \in \Delta \mid d(x, y)=d(x, z)+d(z, y)\}$. As usual, we replace a square bracket by a round bracket if the corresponding endpoint is left out.

Proposition 1.4 If $d(x, y)<\pi$, then $[x, y]$ lies in any apartment that contains $x$ and $y$. Therefore $[x, y]$ is the unique segment joining $x$ and $y$. If $d(x, y)=\pi$ then $[x, y]$ is the union of apartments containing $x$ and $y$.

According to Bridson and Haefliger [10, II.1 Proposition 1.4 (1)], there are deformations along geodesic segments for spherical buildings.

Proposition and Definition 1.5 For $x, y \in \Delta$ with $d(x, y)<\pi$ and $t \in[0,1]$ let $r_{\Delta}(x, y, t) \in[x, y]$ be the point defined by $d\left(x, r_{\Delta}(x, y, t)\right)=t d(x, y)$. The map

$$
r_{\Delta}:\{(x, y) \in \Delta \times \Delta \mid d(x, y)<\pi\} \times[0,1] \rightarrow \Delta ;(x, y, t) \mapsto r_{\Delta}(x, y, t)
$$

is continuous with respect to the metric topology.

By the uniqueness of $d$, the apartments of $\Delta$ are spheres, triangulated by the hyperplanes of a finite essential reflection group, since finite Coxeter complexes can be realized this way. It is clear that roots are closed hemispheres and that walls are the corresponding equators. Hence, $\sigma, \tau \in \mathcal{S}(\Delta)$ are opposite, if and only if there are points $x \in \sigma$ and $y \in \tau$ with $d(x, y)=\pi$. Two points at distance $\pi$ are called antipodal. 
Notation 1.6 For a point $x \in \Delta$, the set of its antipodal points will be denoted by $\operatorname{Ant}(x)$. Furthermore we denote $\operatorname{Ant}^{*}(x)=\operatorname{Ant}(x) \cup\{x\}$.

A subset of $\Delta$ is $\pi$-convex if it contains the joining segments for every pair of nonantipodal points out of it. By Tits [26, Theorem 2.19] a subcomplex of $\Delta$ is convex in the sense of $[26,1.5]$, if and only if its intersection with any apartment is an intersection of roots, that is, if and only if its intersection with any apartment is $\pi$-convex. Then it is also $\pi$-convex as a subset, since by Proposition 1.4 a subset is $\pi$-convex, if and only if its intersection with any apartment is $\pi$-convex.

For simplicity, we call a subset of $\Delta$ convex if it is $\pi$-convex. The complement of a convex set is said to be coconvex.

For a set of simplices $M \subseteq \mathcal{S}(\Delta)$, we denote the full convex hull in the sense of Tits $[26,1.5]$ by $\operatorname{Conv}(M)$. Note, that this differs in general from the metric convex hull.

Notation 1.7 If $\sim$ is one of the relations $<, \leq,>, \geq$, or $=$ and $x \in \Delta$, we put $\Omega_{\Delta}^{\sim}(x)=\{y \in \Delta \mid d(x, y) \sim \pi / 2\}$.

Lemma 1.8 Let $x \in \Delta$ be a point. The balls $\Omega_{\Delta}^{<}(x)$ and $\Omega_{\Delta}^{\leq}(x)$ are convex.

Proof Let $y, z \in \Delta$ be non-antipodal points and let $v \in[y, z]$. By Proposition 1.4, there is an apartment $\Sigma$ containing $[y, z]$. Let $\rho$ be the retraction onto $\Sigma$ centered at some chamber of $\Sigma$ containing $v$ in its closure. Then $d(y, \rho(x)) \leq d(y, x), d(z, \rho(x)) \leq$ $d(z, x)$ and $d(v, \rho(x))=d(v, x)$ hold by Proposition 1.2. Therefore, the lemma follows from the convexity of hemispheres in apartments.

Proposition and Definition 1.9 Let $x \in \Delta$ be a point. For $y \in \Delta \backslash \operatorname{Ant}^{*}(x)$ exists a unique point $p_{x} y \in \partial \mathrm{St} x$ such that $p_{x} y \in[x, y]$ or $y \in\left[x, p_{x} y\right]$. The geodesic projection

$$
p_{x}: \Delta \backslash \operatorname{Ant}^{*}(x) \longrightarrow \partial \mathrm{St} x ; y \longmapsto p_{x} y
$$

with center $x$ onto the boundary of St $x$ is continuous with respect to the metric topology.

Proof The map $p_{x}$ is well defined by Proposition 1.4. The continuity follows from Proposition 1.2 since the restriction $\left.p_{x}\right|_{\Sigma}$ is continuous for any apartment $\Sigma$ that contains $x$. 
For a simplex $\sigma \in \mathcal{S}(\Delta)$, we denote the projection to the star of $\sigma$ in the sense of Tits [26, 2.30] by $\operatorname{proj}_{\sigma}$. By definition, $\operatorname{proj}_{\sigma} \tau$ is the maximal simplex of $\operatorname{St} \sigma \cap \operatorname{Conv}(\sigma, \tau)$. The geodesic projection and the combinatorial projection are related by the following lemma.

Lemma 1.10 Let $x \in \Delta$ and $y \in \Delta \backslash \operatorname{Ant}^{*}(x)$ be points. Let $\sigma$ and $\tau$ be the simplices carrying $x$ and $y$, respectively. Then $\left(x, p_{x} y\right)$ is contained in $\operatorname{proj}_{\sigma} \tau$.

For $x \in \Delta$ and $y, z \in \Delta \backslash \operatorname{Ant}^{*}(x)$ let $\angle_{x}(y, z)$ denote the angle of the triangle $(x, y, z)$ at $x$. Since links are spherical buildings and the canonical metric is unique, we get the following lemma from Charney and Lytchak [17, Proposition 2.3 (2)].

Lemma 1.11 The canonical metric on the link of a vertex $x$ is given by $L_{x}$.

The spherical law of cosines (see Bridson and Haefliger [10, 1.2 Proposition 2.2]) relates the length of a side in a spherical triangle to its opposite angle. Using 1.2, geodesic projection and additionally Proposition 1.4 for the "only if"-part, one gets:

Proposition 1.12 (Spherical law of cosines) Let $x$ be a point of $\Delta$ and let $y, z$ be points of $\Delta \backslash \operatorname{Ant}^{*}(x)$. Then:

$$
\cos d(y, z) \leq \cos d(x, y) \cos d(x, z)+\sin d(x, y) \sin d(x, z) \cos \angle_{x}(y, z)
$$

Equality holds if and only if $x, y$ and $z$ are contained in an apartment.

If $\Delta=\Delta_{1} * \Delta_{2}$ is a reducible spherical building, then $\Delta$ is a spherical join, that is, the inclusions $\Delta_{k} \subset \Delta$ are isometric embeddings and the distance of points lying in different factors is $\pi / 2$. Hence, chambers of reducible spherical buildings contain points at distance $\pi / 2$.

Lemma 1.13 The length of edges joining two vertices does not exceed $\pi / 2$.

Proof We use induction on $\operatorname{dim} \Delta$. The case $\operatorname{dim} \Delta=1$ is clear. Suppose $\operatorname{dim} \Delta>1$. Let $x, y$ and $z$ be vertices of a common chamber. By the induction hypothesis and Lemma 1.11 the angles $\angle_{x}(y, z), \angle_{y}(x, z)$ and $\angle_{z}(x, y)$ of the triangle $(x, y, z)$ are not obtuse. We therefore get the assertion, since the edges of a spherical triangle without obtuse angles can not be longer than $\pi / 2$.

Corollary 1.14 The diameter of closed chambers does not exceed $\pi / 2$. 
Proposition 1.15 Let $C$ be a chamber of $\Delta$ and $x, y \in \bar{C}$. If $d(x, y)=\pi / 2$, then $\Delta=\Delta_{x} * \Delta_{y}$ is a spherical join, such that $x \in \Delta_{x}$ and $y \in \Delta_{y}$. That is, a spherical building is reducible if and only if there is a chamber containing points at distance $\pi / 2$.

Proof The assertion follows immediately from the Buekenhout product theorem [12, Theorem 7.3], once we show that $C$ has two complementary faces $\sigma$ carrying $x$ and $\tau$ carrying $y$, such that $d(u, v)=\pi / 2$, for all $u \in \operatorname{vt}(\sigma)$ and $v \in \operatorname{vt}(\tau)$.

Let $u$ be some vertex of $C$. By Corollary 1.14 we get $d(x, u) \leq \pi / 2$ and $d(y, u) \leq \pi / 2$. Furthermore $L_{u}(x, y)$ does not exceed $\pi / 2$ by Corollary 1.14 and Lemma 1.11. Hence, using the spherical law of cosines on the triangle $(x, u, y)$ we obtain $d(x, u)=\pi / 2$ or $d(y, u)=\pi / 2$.

Now let $\sigma$ be the face of $C$ whose vertices have distance less than $\pi / 2$ to $x$ and let $\tau=C \backslash \sigma$ be the complementary face. Then $x$ lies in $\bar{\sigma}$ and $y$ is a point of $\bar{\tau}$, because the distances from a point to the vertices of the simplex carrying that point are less than $\pi / 2$. For $u \in \operatorname{vt}(\sigma)$ and $v \in \operatorname{vt}(\tau)$, we know that $d(x, v)=\pi / 2, d(x, u)<\pi / 2$ and $\angle_{u}(x, v) \leq \pi / 2$. Hence, $d(v, u)=\pi / 2$ by the spherical law of cosines.

\section{Coconvex supported subcomplexes}

Definition 2.1 Let $\Lambda$ be a simplicial complex and let $M$ be a subset of $\Lambda$. By $\Lambda(M)$ we denote the maximal subcomplex of $\Lambda$ contained in $M$. We shorten $\Lambda^{\prime}(M)=$ $\Lambda^{\prime}\left(M \cap \Lambda^{\prime}\right)$ for a subcomplex $\Lambda^{\prime} \subseteq \Lambda$. The set $M$ is said to be a support of $\Lambda(M)$. A subcomplex $\Lambda^{\prime} \subseteq \Lambda$ is a (P) supported subcomplex (of $\Lambda$ ) if and only if $\Lambda^{\prime}$ admits a support with property $(\mathrm{P})$.

We investigate the connectedness properties of coconvex supported subcomplexes of spherical buildings. This section is dedicated to the proof of the first main result.

Theorem A Non-empty, closed, coconvex supported subcomplexes of spherical buildings are homotopy Cohen-Macaulay. They are non-contractible for at least onedimensional, thick buildings.

The first claim is shown in Proposition 2.5 and Corollary 2.7; the second claim is Proposition 2.8.

Note that coconvex supported subcomplexes are not coconvex in general. But for a coconvex set $M$ and a simplex $\sigma$ which is not contained in neither $M$ nor its complement, $M \cap \partial \sigma$ is a strong deformation retract of $M \cap \bar{\sigma}$. We can therefore 
construct a sequence $\Lambda=\Lambda_{n} \supseteq \cdots \supseteq \Lambda_{0}=\Lambda(M)$ of subcomplexes such that the maximal dimension of simplices like $\sigma$ is decreasing and $\Lambda_{i} \cap M$ is a strong deformation retract of $\Lambda_{i+1} \cap M$. This means that coconvex supported subcomplexes are homotopy equivalent to their coconvex supports. We record this observation.

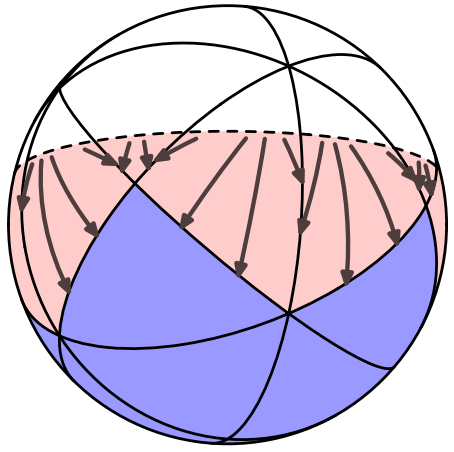

(a)

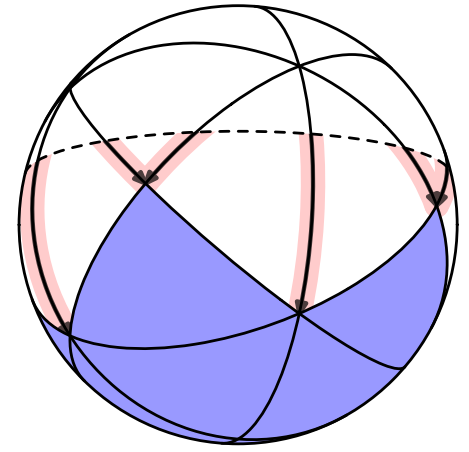

(b)

Figure 1: Deformation of a coconvex support onto the supported subcomplex

Observation 2.2 Let $\Lambda$ be a subcomplex and let $M$ be a coconvex subset of $\Delta$. Then $\Lambda \cap M$ and $\Lambda(M)$ are homotopy equivalent.

Lemma 2.3 Suppose $\operatorname{dim} \Delta>0$. If $M$ is an open, convex subset of $\Delta$ containing a pair of antipodes, then $M=\Delta$.

Proof Let $x, y \in M$ be antipodal points, contained in some apartment $\Sigma$. Then $\Sigma$ is contained in $M$, since the convex hull of $x$ together with a neighborhood of $y$ covers $\Sigma$. The closure of any chamber $C$ that intersects $M$ is contained in $M$, since $C$ has an opposite chamber in $\Sigma$ and we therefore get an apartment in $M$ (as above) containing both. Now the assertion follows by induction on the gallery distance from $\Sigma$.

Next we will show that closed, coconvex supported subcomplexes are spherical. The proof is mainly based on the following lemma.

Lemma 2.4 (von Heydebreck [19, Lemma 3.5]) Let $C$ be chamber of $\Delta$ and let $M$ be a finite set of apartments containing $C$. Then there is a finite set $M^{\prime}=\left\{\Sigma_{1}, \ldots, \Sigma_{r}\right\}$ of apartments containing $C$ with $M \subseteq M^{\prime}$ such that $\Sigma_{j} \cap\left(\bigcup_{i=1}^{j-1} \Sigma_{i}\right)$ is a union of half-apartments containing $C$ for $2 \leq j \leq r$.

Proposition 2.5 Closed, coconvex supported subcomplexes of $\Delta$ are $\operatorname{dim} \Delta$-spherical or empty. 
Proof Let $M$ be a non-empty, closed and coconvex subset of $\Delta$. Since the case $\operatorname{dim} \Delta=0$ is trivial and the case $\Delta=\Delta(\Delta)$ is covered by the Solomon-Tits theorem, suppose that $M$ is a proper subset and $\operatorname{dim} \Delta>0$.

Let $\Sigma$ be an apartment whose intersection with $M$ is not empty. Then $\Sigma \backslash M$ is contained in an open hemisphere of $\Sigma$, since it is a proper, open, convex subset. Therefore $\Sigma \cap M$ contains a closed chamber by Corollary 1.14. Hence, $\Delta(M)$ is $\operatorname{dim} \Delta$-dimensional.

Let $C$ be a chamber not contained in $M$. Any finite subcomplex of $\Delta(M)$ is coverable by a finite set $\left\{\Sigma_{1}, \ldots, \Sigma_{m}\right\}$ of apartments, each of which contains $C$. Let us denote $\Lambda_{r}=\Sigma_{1} \cup \ldots \cup \Sigma_{r}$ and $\Psi_{r}=\Sigma_{r} \cap \Lambda_{r-1}$. According to Lemma 2.4 we may choose the set of apartments and their order such that, for any $r, \Psi_{r}$ is a union of roots in $\Sigma_{r}$. We prove the $(\operatorname{dim} \Delta-1)$-connectedness of $\Lambda_{m}(M)$. Clearly, as $\Sigma_{r} \cap M$ is closed and coconvex, $\Sigma_{r}(M) \approx \Sigma_{r} \cap M$ is $(\operatorname{dim} \Delta-1)$-connected. Hence, the desired assertion follows from Lemma 1.1(a), once we show that $\Psi_{r} \cap M \approx \Psi_{r}(M)=$ $\Sigma_{r}(M) \cap \Lambda_{r-1}(M)$ is $(\operatorname{dim} \Delta-2)$-connected.

Let $x$ be a point of $C \backslash M$ and let $p: \Sigma_{r} \backslash \operatorname{Ant}^{*}(x) \rightarrow \partial\left(\Sigma_{r} \cap M\right)$ denote the geodesic projection with center $x$ onto the boundary of $\Sigma_{r} \cap M$. Since $\Psi_{r}$ is a union of roots, each of which contains $x$ as an inner point, we know that $\Psi_{r}$ is star shaped with respect to $x$ and does not contain the antipode of $x$ in $\Sigma_{r}$. Therefore the restriction of $p$ to $\Psi_{r} \cap M$ is a retraction $\Psi_{r} \cap M \rightarrow \Psi_{r} \cap \partial\left(M \cap \Sigma_{r}\right)$ inducing a strong deformation retraction

$$
\left(\Psi_{r} \cap M\right) \times[0,1] \longrightarrow \Psi_{r} \cap M ;(z, t) \longmapsto r_{\Delta}(z, p(z), t)
$$

from $\Psi_{r} \cap M$ onto $\Psi_{r} \cap \partial\left(M \cap \Sigma_{r}\right)$. Hence, $\Psi_{r} \cap M \approx \Psi_{r} \cap \partial\left(M \cap \Sigma_{r}\right)$.

Observe that $p$ maps $\left(\Sigma_{r} \backslash M\right) \backslash \Psi_{r}$ onto the complement $\partial\left(M \cap \Sigma_{r}\right) \backslash \Psi_{r}$ of the above retract. Let $q: \Sigma_{r} \backslash \operatorname{Ant}^{*}(x) \rightarrow \Omega_{\Sigma_{r}}(x)$ denote the geodesic projection with center $x$ onto the equator $\Omega_{\Sigma_{r}}(x)$. Note that the restriction of $q$ to $\partial\left(M \cap \Sigma_{r}\right)$ is the inverse homeomorphism of $\left.p\right|_{\Omega_{\Sigma_{r}}(x)}$ and that $q=q \circ p$. Furthermore $q$ maps open, convex subsets of $\Sigma_{r} \backslash \operatorname{Ant}^{*}(x)$ to open, convex sets. Since $\left(\Sigma_{r} \backslash M\right) \backslash \Psi_{r}$ is open and convex, $q\left(\partial\left(M \cap \Sigma_{r}\right) \backslash \Psi_{r}\right)=q\left(\left(\Sigma_{r} \backslash M\right) \backslash \Psi_{r}\right)$ is an open, convex subset of $\Omega_{\Sigma_{r}}^{=}(x)$. Therefore $\Psi_{r} \cap \partial\left(M \cap \Sigma_{r}\right)$ is $(\operatorname{dim} \Delta-2)$-connected, since it is the homeomorphic image of a closed, coconvex subset of $\Omega_{\Sigma_{r}}^{=}(x)$.

Remark K-U Bux, R Köhl and S Witzel observed the assumptions of Proposition 2.5 could be weakened, since its proof mainly used that the intersections of $M$ with apartments containing $C$ are coconvex, but not that $M$ is coconvex. So they showed in [16, Proposition 4.3] that a complex is spherical provided that it is supported by a set that has a coconvex intersection with any apartment containing a given chamber. 


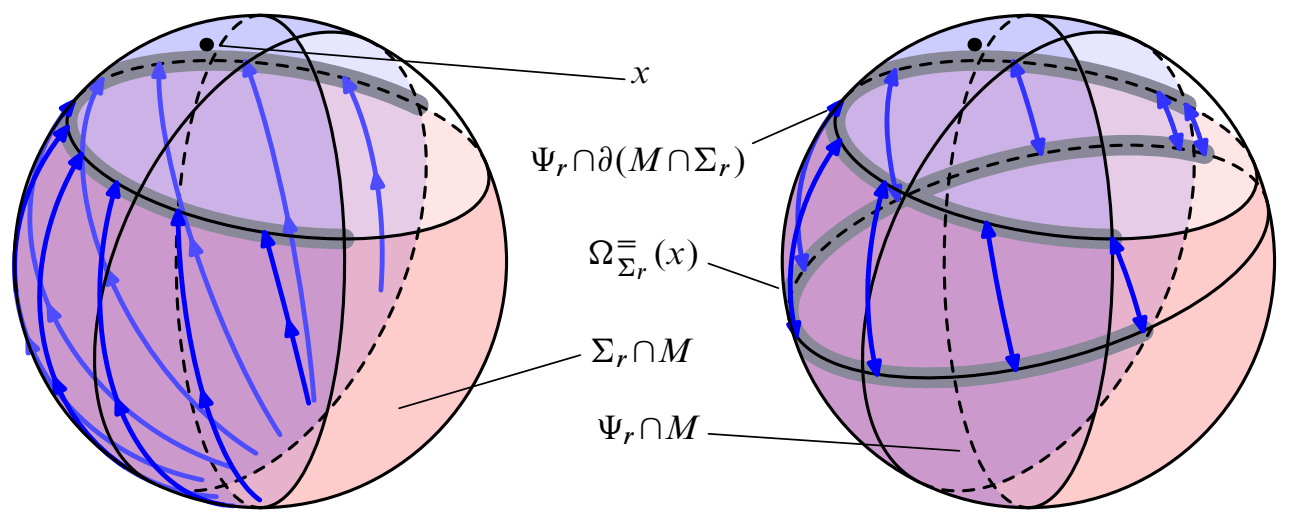

(a)

(b)

Figure 2: Via geodesic projection with center $x$ we get (a) a homotopy equivalence $\Psi_{r} \cap M \approx \Psi_{r} \cap \partial\left(M \cap \Sigma_{r}\right)$ and (b) a homeomorphism of $\Psi_{r} \cap \partial\left(M \cap \Sigma_{r}\right)$ and a coconvex subset of $\Omega_{\Sigma_{r}}(x)$

Lemma 2.6 Suppose $\operatorname{dim} \Delta>0$. Let $x, y \in \Delta$ be opposite vertices and let $M$ be a proper, open, convex subset of $(x, y)$ (see Notation 1.3 and Proposition 1.4). Then the image of $M$ under the geodesic projection onto Lk $x$ is a proper, open, convex subset of $\operatorname{Lk} x$.

Proof Let $A$ be an arbitrary apartment of $\operatorname{Lk} x$. The convex hull of $x, y$ and $A$ is an apartment $\Sigma$. For a point $z \in A$, the geodesic segment joining $x$ and $y$ going through $z$ is contained in $\Sigma$. Therefore $\Sigma$ contains the preimage of $z$ under the restriction $\left.p_{x}\right|_{(x, y)}$. Hence, $p_{x}(M) \cap A=p_{x}(M \cap \Sigma)$.

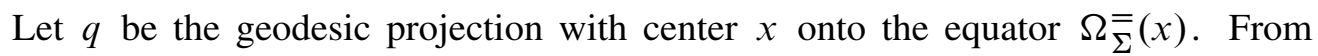
$q=\left.q \circ p_{x}\right|_{\Sigma}$ we get $q(M \cap \Sigma)=q\left(p_{x}(M) \cap A\right)$. Therefore $q\left(p_{x}(M) \cap A\right)$ is an open, convex subset of $\Omega_{\Sigma}^{\bar{\Sigma}}(x)$, because $q$ maps open, convex subsets of $\Sigma \backslash$ Ant $^{*}(x)$ to open, convex subsets of $\Omega_{\Sigma}^{\bar{E}}(x)$. Since the restriction of $q$ on $A$ is an isometry according to Lemma $1.11, p_{x}(M) \cap A$ is open and convex in Lk $x$. Recall that $A$ has been chosen arbitrary. Hence, $p_{x}(M)$ is open and convex in Lk $x$.

Assume there are $u, v \in M$ such that $\angle_{x}\left(p_{x} u, p_{x} v\right)=\pi / 2$. Then the union of segments $[x, u] \cup[u, y] \cup[y, v] \cup[v, x]$ would be a great circle. But this is impossible, because $M$ would contain $x$ or $y$ or a pair of antipodal points from $\Delta$. Hence, $p_{x}(M)$ is a proper subset of $\operatorname{Lk} x$.

Corollary 2.7 The links in non-empty, closed, coconvex supported subcomplexes of $\Delta$ are non-empty, closed, coconvex supported subcomplexes. 
Proof It is sufficient to prove the assertion for vertices. Let $M \subset \Delta$ be a proper, open, convex subset and let $x$ be a vertex in $\Delta \backslash M$. Since $M$ is open, a simplex of Lk $x$ is contained in the link $\operatorname{Lk}_{\Delta(\Delta \backslash M)} x$ if and only if its closure does not intersect the image $p_{x}(M \cap$ St $x)$. Let $y$ op $x$ be a vertex. Since $[x, y]$ contains a neighborhood of $x$, by Kleiner and Leeb [21, Lemma 3.6.1], it also contains St $x$. Hence, the assertion follows from Lemma 2.6.

Proposition 2.8 Non-empty, closed, coconvex subsets of at least one-dimensional, thick spherical buildings are non-contractible.

Proof Let $\Delta$ be at least one-dimensional and let $M \subset \Delta$ be a proper, open, convex subset. We proof the existence of a $\operatorname{dim} \Delta$-dimensional sphere in $\Delta \backslash M$ by induction on $\operatorname{dim} \Delta$.

Since $\Delta$ is thick, there are three pairwise opposite chambers. Then there is a pair $x$ op $y$ of opposite vertices inside $\Delta \backslash M$ by Lemma 2.3.

Let $S$ be the union of the open geodesic segments that join $x, y$ and contain a point from $M^{\prime}=p_{x}(M \cap[x, y])$. Then $[x, y] \backslash S$ is a subset of $\Delta \backslash M$. Furthermore $[x, y] \backslash S$ is the spherical join of $\{x, y\}$ and $\left(\operatorname{Lk} x \backslash M^{\prime}, L_{x}\right)$ by Kleiner and Leeb [21, Proposition 3.10.1]. Hence we are done, if $\operatorname{Lk} x \backslash M^{\prime}$ contains a $(\operatorname{dim} \operatorname{Lk} x)-$ dimensional sphere. Clearly, that is assured by the induction hypothesis, provided that $\operatorname{dim} \Delta>1$, since $M^{\prime}$ is a proper, open, convex subset of $\operatorname{Lk} x$ by Lemma 2.6. But even if $\operatorname{dim} \Delta=1$ we get a 0 -sphere in $\operatorname{Lk} x \backslash M^{\prime}$, since $M^{\prime}$ is connected and Lk $x$ is thick.

\section{Hemisphere complexes}

In this section we will examine some special coconvex supported subcomplexes. Their supports are unions of hemispheres, so the complexes will be called hemisphere complexes. Throughout the remainder of this paper we fix an arbitrary point $x$ of $\Delta$.

Definition 3.1 The subcomplex $\Delta^{>}(x)=\Delta\left(\Omega_{\Delta}^{>}(x)\right)$ is said to be the open hemisphere complex of $\Delta$ with respect to the pole $x$ and $\Delta^{\geq}(x)=\Delta\left(\Omega_{\Delta}^{\geq}(x)\right)$ is said to be the closed hemisphere complex of $\Delta$ with respect to the pole $x \cdot \Delta^{=}(x)=\Delta\left(\Omega_{\Delta}^{=}(x)\right)$ is the equator complex of $x$.

The sets $\Omega_{\Delta}^{<}(x)$ and $\Omega_{\Delta}^{\leq}(x)$ are convex by Lemma 1.8. Hence, hemisphere complexes are coconvex supported subcomplexes. 
Corollary 3.2 Closed hemisphere complexes of thick spherical buildings are homotopy Cohen-Macaulay and non-contractible.

Proof The assertion is an immediate consequence of Theorem A except for the noncontractibility in the case $\operatorname{dim} \Delta=0$. But closed hemisphere complexes are also in this case non-contractible, since $\Delta$ is thick and $\Omega_{\Delta}^{<}(x)$ is a single point.

Note that the intersection of $\Omega_{\Delta}^{\sim}(x)$ with apartments containing $x$ is convex. Hence, $\Omega_{\Delta}(x) \cap \sigma$ is convex for any simplex $\sigma$. We therefore get the following observation.

Observation 3.3 Open hemisphere complexes, closed hemisphere complexes and equator complexes are full subcomplexes of $\Delta$.

Notation 3.4 If $\Delta$ is reducible, then $\Delta_{\text {hor }}(x)$ denotes the maximal join factor of $\Delta$ that is contained in $\Omega_{\Delta}^{=}(x)$ and $\Delta_{\text {ver }}(x)$ denotes the minimal join factor containing $x$.

We certainly have $\Delta=\Delta_{\text {hor }}(x) * \Delta_{\text {ver }}(x)$, since any irreducible join factor that does not intersect the closure of the simplex carrying $x$ lies in $\Omega_{\Delta}(x)$ by Proposition 1.15. Now let us have a look at the induced join decomposition of hemisphere complexes:

Let $\Delta=\Delta_{1} * \Delta_{2}$ be a reducible spherical building and let $\sim$ be one of the relations $>$, $\geq$, or $=$. We get $\Delta^{\sim}(x)=\Delta_{1}\left(\Omega_{\Delta}^{\sim}(x)\right) * \Delta_{2}\left(\Omega_{\Delta}^{\sim}(x)\right)$ from Observation 3.3. If $x$ is a point of $\Delta_{1}$, then $\Delta_{1}\left(\Omega_{\Delta}^{>}(x)\right)=\Delta^{>}(x)$ and $\Delta_{2}$ is a subcomplex of $\Delta_{\text {hor }}(x)$. Therefore $\Delta_{2}\left(\Omega_{\Delta}^{\sim}(x)\right)$ is empty if $\sim$ is a strong inequality or all of $\Delta_{2}$ otherwise. If $x$ is not contained in neither $\Delta_{1}$ nor $\Delta_{2}$ then there are two unique points $x_{1} \in \Delta_{1}$ and $x_{2} \in \Delta_{2}$ such that $x$ lies inside their joining segment. In this case we have $\Delta_{i}\left(\Omega_{\Delta}^{\sim}(x)\right)=\Delta_{i}^{\sim}\left(x_{i}\right)$ :

Assume $\{i, j\}=\{1,2\}$ and $y \in \Delta_{i}$. Then $d\left(x_{1}, x_{2}\right)=\pi / 2=d\left(y, x_{j}\right)$, since points of disjoint factors have distance $\pi / 2$. There is an apartment containing $x, x_{1}, x_{2}$ and $y$. We therefore get from the spherical law of cosines

$$
\begin{aligned}
\cos d(x, y) & =\sin d\left(x_{j}, x\right) \cos \angle_{x_{j}}(x, y) \\
& =\sin d\left(x_{j}, x\right) \cos \angle_{x_{j}}\left(x_{i}, y\right) \\
& =\sin d\left(x_{j}, x\right) \cos d\left(x_{i}, y\right) .
\end{aligned}
$$

Hence, $d(x, y) \sim \pi / 2$ if and only if $d\left(x_{i}, y\right) \sim \pi / 2$. We proved:

Proposition 3.5 Assume $\Delta_{\mathrm{ver}}(x)=\Delta_{1} * \cdots * \Delta_{k}$ is a decomposition of $\Delta_{\mathrm{ver}}(x)$ into irreducible factors. Then $\Delta^{>}(x)=\Delta_{\text {ver }}^{>}(x)=\Delta_{1}\left(\Omega_{\Delta}^{>}(x)\right) * \cdots * \Delta_{k}\left(\Omega_{\Delta}^{>}(x)\right)$ is a join of open hemisphere complexes in $\Delta_{1}, \ldots, \Delta_{k}$ and the equator complex decomposes to $\Delta^{=}(x)=\Delta_{\text {ver }}^{=}(x) * \Delta_{\text {hor }}(x)$. 
If $\Delta$ is irreducible, then the closed stars of the simplices opposite to the simplex carrying $x$ are contained in $\Omega_{\Delta}^{>}(x)$, since the diameter of closed chambers is less than $\pi / 2$ by Proposition 1.15. Hence, open hemisphere complexes of irreducible spherical buildings have the same dimension as the surrounding building. In general, $\operatorname{dim} \Delta^{>}(x)=\operatorname{dim} \Delta_{\text {ver }}(x) \leq \operatorname{dim} \Delta$ by Proposition 3.5; and the last inequality is strict if $\Delta_{\text {hor }}(x)$ is not empty. In the sequel we will have to take care of this case.

Lemma and Definition 3.6 Let $\sigma$ be a simplex of $\Delta^{=}(x)$. There is a unique point $p_{\sigma} x \in \operatorname{Lk} \sigma$ such that

$$
d(x, y) \sim \pi / 2 \Longleftrightarrow d_{\mathrm{Lk} \sigma}\left(p_{\sigma} x, y\right) \sim \pi / 2,
$$

for any point $y \in \mathrm{Lk} \sigma$ and any relation $<, \leq,=, \geq$ or $>$. For the simplex $\xi$ carrying $x$ and the simplex $\chi$ carrying $p_{\sigma} x$, holds $\sigma \cup \chi=\operatorname{proj}_{\sigma} \xi$. (If $\sigma$ is a vertex then $p_{\sigma} x$ is the geodesic projection of $x$ on $\partial \operatorname{St} \sigma=\operatorname{Lk} \sigma$.)

Proof We induct on the dimension of $\sigma$. So let $\sigma \in \Delta^{=}(x)$ be a vertex and let $y \in \operatorname{Lk} \sigma$ be a point. There is an apartment containing $x, y$ and $\sigma$. By the spherical law of cosines and Lemma 1.11 we therefore get

$$
\cos d(x, y)=\sin d(\sigma, y) \cos \angle_{\sigma}(x, y)=\sin d(\sigma, y) \cos d_{\mathrm{Lk} \sigma}\left(p_{\sigma} x, y\right) .
$$

Hence, $d(x, y) \sim \pi / 2$ if and only if $d_{\operatorname{Lk} \sigma}\left(p_{\sigma} x, y\right) \sim \pi / 2$. The assertion on the projection is an immediate consequence of Lemma 1.10 .

For simplices $\sigma$ of higher dimension, one obtains the assertion and the characterization of $p_{\sigma} x$ by regarding a simplex as a vertex in the link of one of its codimension-1-faces. This is justified, since $\operatorname{proj}_{\sigma} \xi=\operatorname{proj}_{\sigma} \operatorname{proj}_{\tau} \xi$ for $\tau \leq \sigma$ by Tits [26, 2.30.5] and since the canonical metric is unique.

Lemma 3.7 The links in open hemisphere complexes of irreducible buildings are non-empty, closed, coconvex supported subcomplexes.

Proof Suppose $\Delta$ is irreducible. Let $\sigma$ be a simplex of $\Delta^{>}(x)$. The idea is to recognize $\operatorname{Lk}_{\Delta^{>}(x)} \sigma$ as a link $\operatorname{Lk}_{\Delta^{\geq}\left(x^{\prime}\right)} \sigma$ in a closed hemisphere complex (to a slightly perturbed pole $x^{\prime}$ ) and to apply Corollary 2.7. The task is to choose $x^{\prime}$.

Let $y$ be a point of $\sigma$. As $D=\left\{|d(x, z)-\pi / 2| \mid z \in \operatorname{vt}(\Delta) \backslash \operatorname{vt}\left(\Delta^{=}(x)\right)\right\}$ is finite by Proposition 1.2, we may chose a point $x^{\prime}$ on a segment joining $x$ and $y$ such that $0<d\left(x, x^{\prime}\right)<\min D$. From the triangle inequality we get the implications $d(x, z)>\pi / 2 \Rightarrow d\left(x^{\prime}, z\right)>\pi / 2$ and $d(x, z)<\pi / 2 \Rightarrow d\left(x^{\prime}, z\right)<\pi / 2$ for any vertex $z$ of $\Delta$. Hence,

$$
\operatorname{vt}\left(\Delta^{>}(x)\right) \subseteq \operatorname{vt}\left(\Delta^{>}\left(x^{\prime}\right)\right) \subseteq \operatorname{vt}\left(\Delta^{\geq}\left(x^{\prime}\right)\right) \subseteq \operatorname{vt}\left(\Delta^{\geq}(x)\right) .
$$


Therefore $\operatorname{Lk}_{\Delta^{>}(x)} \sigma$ is contained in $\operatorname{Lk}_{\Delta^{\geq}\left(x^{\prime}\right)} \sigma$. Let $z$ be a vertex of $\operatorname{Lk}_{\Delta^{\geq}\left(x^{\prime}\right)} \sigma$. According to Proposition 1.15, $d(y, z)$ is less than $\pi / 2$. If $y$ is an antipode of $x$, then we have $d(x, z)>\pi / 2$. If $x$ and $y$ are not antipodal, we deduce $d(x, z)>d\left(x^{\prime}, z\right)$ from $d(x, y)>d\left(x^{\prime}, y\right)>\pi / 2, L_{y}(x, z)=\angle_{y}\left(x^{\prime}, z\right)$ and the spherical law of cosines. Hence, $\operatorname{Lk}_{\Delta^{>}(x)} \sigma=\operatorname{Lk}_{\Delta^{\geq}\left(x^{\prime}\right)} \sigma$, because their vertex sets coincide. Now the lemma follows from Corollary 2.7.

The following proposition, together with the results we previously reached immediately imply our second main result.

Proposition 3.8 Let $\Delta$ be a thick spherical building. Then $\Delta^{>}(x)$ is $\operatorname{dim} \Delta_{\text {ver }}(x)-$ spherical and non-contractible.

In particular:

Theorem B Open hemisphere complexes of thick spherical buildings are homotopy Cohen-Macaulay and non-contractible.

Proof By Proposition 3.8 the open hemisphere complex $\Delta^{>}(x)$ is $\operatorname{dim} \Delta_{\text {ver }}(x)-$ spherical and non-contractible. Let $\sigma$ be a simplex of $\Delta^{>}(x)$. From Proposition 3.5 and Lemma 3.7 we know that irreducible join factors of $\Delta_{\text {ver }}(x)$ containing a non-empty face of $\sigma$, intersect $\operatorname{Lk} \sigma$ in a non-empty, closed, coconvex supported subcomplex. The intersection of $\operatorname{Lk} \sigma$ with an irreducible join factor of $\Delta_{\text {ver }}(x)$ that does not meet $\sigma$ is an open hemisphere complex according to Proposition 3.5. Then $\operatorname{Lk}_{\Delta^{>}(x)} \sigma$ is a $\left(\operatorname{dim} \Delta_{\text {ver }}(x)-\operatorname{dim} \sigma-1\right)$-dimensional join of open hemisphere complexes and nonempty, closed, coconvex supported subcomplexes. Since its join factors are spherical by Propositions 2.5 and 3.8, so is $\operatorname{Lk}_{\Delta^{>}(x)} \sigma$.

The proof of Proposition 3.8 will occupy the remainder of this paper. Note that the proof of Proposition 2.5 would not work in the case of open hemisphere complexes, since the intersection of an open hemisphere with a union of closed hemispheres is not $(\operatorname{dim} \Delta-2)$-connected in general. A closer look at the proof of Lemma 2.4 (von Heydebreck [19, Lemma 3.5]) suggests that such situations arise inevitably. For classical buildings one is able to achieve a precise description of the links in hemisphere complexes. Therefore I tried to mimic the sphericity proofs Abels and Abramenko used in [2] and [4], but I was not able to avoid limitations on the thickness of the underlying buildings. This led to a different approach: Starting with a closed hemisphere complex, which is known to be spherical by Corollary 3.2, we delete the stars of simplices contained in the equator complex by a filtration such that the boundary of the deleted 


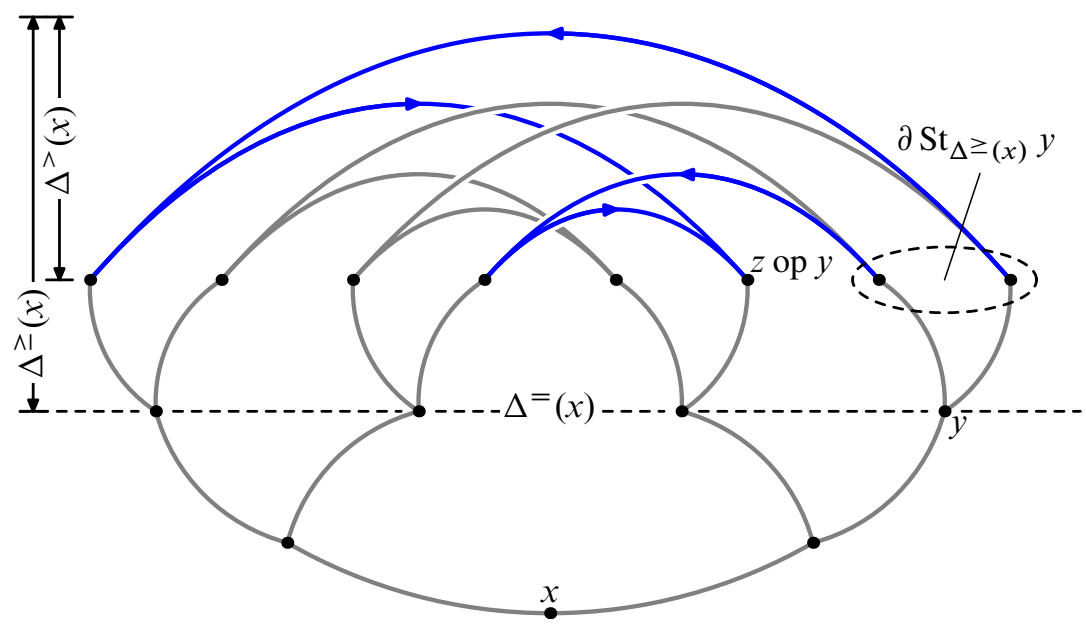

Figure 3: The segments joining $\partial \mathrm{St}_{\Delta \geq(x)} y$ with $z$ op $y$ are contained in $\Delta^{>}(x)$. Hence, the boundary of the $\mathrm{St}_{\Delta^{\geq}(x)} y$ is contractible in $\Delta^{>}(x)$. (Here $\Delta$ is the flag complex of the projective plane over $\mathbb{F}_{2}$.)

stars is contractible in the remaining subcomplex (see Figure 3). To do this, we will have to spend some work in advance. We begin by describing the obstacles that need to be overcome:

For a point $y$ of the equator complex, suppose there is an antipode $z \in \Delta^{>}(x)$. (In the sequel we will show that such an antipode exists.) Any point $u \in \partial \mathrm{St}_{\Delta \geq(x)} y$ is connected to $z$ by a unique geodesic segment. In an ideal world, we could therefore contract $\partial \operatorname{St}_{\Delta^{\geq}(x)} y$ inside $\Omega^{>}(x)$ by geodesically coning off from $z$. This idea works sometimes, but if $u$ lies also in the equator, we would like to see $(u, z] \subseteq \Omega_{\Delta}^{>}(x)$. This, however, does not always happen. There are two obstructions.

Obstruction 3.9 Let $\sigma$ be the simplex carrying $y$ and let $\tau$ denote the simplex carrying $u$. As $(u, z]$ is contained in a geodesic segment joining $y$ and $z$, the initial segment $(u, z] \cap \operatorname{St} \tau$ of $(u, z]$ lies in a simplex $\theta$ of St $\tau$ that is opposite to $\sigma \cup \tau$ in St $\tau$. If $\sigma \backslash \tau$ is a simplex of a join factor of $\operatorname{Lk} \tau$ that lies in $\Omega=\bar{\Delta}(x)$, then $\theta$ is contained in the equator, since $\theta \backslash \tau$ is also a simplex of that join factor. In this case $(u, z]$ can not be contained in $\Omega_{\Delta}^{>}(x)$ regardless of which antipode $z$ of $y$ we use.

Our first step will be to circumvent this problem. In Section 4 we construct a filtration that removes $\tau$ from the equator complex before $\sigma$ is removed, if $\sigma \cup \tau$ is a simplex of the equator complex and $\sigma \backslash \tau$ is contained in a join factor of $\operatorname{Lk} \tau$ that lies in $\Omega_{\Delta}^{=}(x)$. 
Obstruction 3.10 Even if we can find an antipode $z$ for any $u \in \partial \operatorname{St}_{\Delta \geq(x)} y$ such that $(u, z] \subseteq \Omega_{\Delta}^{>}(x)$, it may happen that there is no antipode $z$ such that the cone over $\partial \mathrm{St}_{\Delta \geq(x)} y$ with tip in $z$ is contained in $\Omega_{\Delta}^{>}(x)$. For instance, this case occurs, if the link of the simplex $\sigma$ carrying $y$ has a join factor that lies in the equator and the corresponding (opposite) join factors of the links of the simplices opposite to $\sigma$ are not completely contained in the hemisphere complex.

The example shows that we can not get around the second obstruction. In general we are only able to contract pieces of $\partial \mathrm{St}_{\Delta \geq(x)} y$ by geodesically coning off inside $\Omega^{>}(x)$. Here we use Lemma 1.1(b). Hence, we are forced to find $\operatorname{dim} \Delta$-spherical subcomplexes of the filtration stages that contain the boundaries of the relative stars. In order to deal with this problem, our second step will be to proof a lemma on $\operatorname{dim} \Delta-$ sphericity of a union of cones over subcomplexes of $\partial$ St $y$. This will be the task of Section 5. In Section 6, we establish a family of subcomplexes of the boundaries of the relative stars and suitable antipodes such that the corresponding cones are contained in $\Omega^{>}(x)$. We finally complete the proof of Proposition 3.8 in Section 7.

\section{A filtration of closed hemisphere complexes}

In this section we construct a filtration of the closed hemisphere complex starting with the corresponding open hemisphere complex. The aim is to control the progress of connectivity properties as the filtration shrinks. Since we intent to use Lemma 1.1(b), it will be appropriate that two consecutive complexes of the filtration differ by a disjoint union of relative stars. In view of Obstruction 3.9 we also require the following property: If the upper bound $\sigma \cup \tau$ of $\sigma, \tau \in \mathcal{S}\left(\Delta^{=}(x)\right)$ exists and $\sigma \backslash \tau$ is contained in a join factor of Lk $\tau$ that lies in $\Omega=\bar{\Delta}(x)$ then $\sigma$ is at a lower stage of the filtration than $\tau$. Later on, we shall see that the filtration satisfying these properties affords the desired control of connectivity properties. In the present section we show its existence.

Proposition 4.1 There is a filtration $\Delta^{>}(x) * \Delta_{\text {hor }}(x)=F_{0} \subset F_{1} \subset \cdots \subset F_{N}$ (see Notation 3.4) of the closed hemisphere complex $F_{N}=\Delta^{\geq}(x)$ that satisfies the following two properties:

(a) For $1 \leq k \leq N$, the complex $F_{k}$ is the disjoint union $F_{k-1} \cup \bigcup_{\sigma \in I_{k}} \mathrm{St}_{F_{k}} \sigma$ for some set of simplices $I_{k} \subseteq \mathcal{S}\left(F_{k}\right) \backslash \mathcal{S}\left(F_{k-1}\right)$.

(b) For $1 \leq k \leq N$, every simplex $\sigma \in I_{k}$ and any simplex $\tau$ of $\Delta^{=}(x) \cap \partial \operatorname{St}_{F_{k}} \sigma$, the horizontal part $(\operatorname{Lk} \tau)_{\text {hor }}\left(p_{\tau} x\right)$ of $\operatorname{Lk} \tau$ does not contain $\sigma \backslash \tau$. 
To explain the further strategy, let us at first suppose that we have got a filtration that satisfies Proposition 4.1(a). We agree that $I_{0}=\{\varnothing\}$ and $F_{-1}=\varnothing$. Then any simplex $\sigma \in \mathcal{S}\left(F_{k}\right) \backslash \mathcal{S}\left(F_{k-1}\right)$ has a unique face $\mathcal{R}(\sigma) \in I_{k}$ such that $\sigma$ is contained in the relative star $\operatorname{St}_{F_{k}} \mathcal{R}(\sigma)$. We can characterize $\mathcal{R}(\sigma)$ as the unique minimal face of $\sigma \in \mathcal{S}\left(F_{k}\right) \backslash \mathcal{S}\left(F_{k-1}\right)$ that is not contained in $\mathcal{S}\left(F_{k-1}\right)$. Hence, there is a map $\mathcal{R}: \mathcal{S}\left(\Delta^{\geq}(x)\right) \rightarrow \bigcup_{k} I_{k}$ that determines all relative stars and a grading on its image that determines all stages of the filtration. In analogy to Björner [8, Definition 1.1] we call $\mathcal{R}$ the restriction map of the filtration.

Our task is to construct a filtration that additionally satisfies Proposition 4.1(b). From the geometric point of view, Proposition 4.1(b) means that the walls bordering the relative stars of the filtration in question must not contain $x$. To receive this feature we initially define the restriction map $\mathcal{R}: \mathcal{S}\left(\Delta^{\geq}(x)\right) \rightarrow \mathcal{S}\left(\Delta^{=}(x)\right)$ and hereby decompose $\Delta^{\geq}(x)$ into the relative stars we would like to have. Of course, one has to show that $\mathcal{R}(\sigma)=\mathcal{R}(\tau)$ for any face $\tau$ of $\sigma$ that contains $\mathcal{R}(\sigma)$ in order to assure that $\mathcal{R}^{-1}(\mathcal{R}(\sigma))$ is the star of $\mathcal{R}(\sigma)$ in some subcomplex.

We also need a grading on the image of the restriction map. Since $\mathcal{R}^{-1}(\mathcal{R}(\sigma))$ shall become a relative star, the restrictions $\mathcal{R}(\tau)$ such that $\mathcal{R}(\sigma) \cup \mathcal{R}(\tau)$ is a simplex of $\mathcal{R}^{-1}(\mathcal{R}(\sigma))$ should be at a lower stage than $\mathcal{R}(\sigma)$. We use this observation to define a partial ordering on $\operatorname{im} \mathcal{R}$. If will turn out that strictly increasing chains have bounded length. We finally obtain the grading on im $\mathcal{R}$ from the length of strictly increasing chains.

Definition 4.2 For a chamber $C \in \operatorname{Ch}(\Delta)$ and a vertex $v$ of $C$ let $C_{v}=C \backslash v$ denote its complementary face. For a simplex $\sigma \in \mathcal{S}(\Delta)$ let $\sigma_{x}^{=}$be its maximal face contained in $\Delta^{=}(x)$. The map $\mathcal{R}_{\Delta}^{x}: \mathcal{S}\left(\Delta^{\geq}(x)\right) \longrightarrow \mathcal{S}\left(\Delta^{=}(x)\right)$ defined by

$$
\operatorname{vt}\left(\mathcal{R}_{\Delta}^{x}(\sigma)\right)=\left\{v \in \operatorname{vt}\left(\sigma_{x}^{=}\right) \mid \exists C \in \operatorname{Ch}\left(\operatorname{St} \sigma_{x}^{=}\right): \operatorname{Lk} C_{v} \nsubseteq \Delta^{=}(x)\right\}
$$

(see Figure 4$)$ is called the $(\Delta, x)$-restriction on $\Delta^{\geq}(x)$.

Lemma 4.3 For $x, y \in \Delta$ the following statements are equivalent:

(a) $\Delta=\Delta_{x} * \Delta_{y}$ decomposes as a spherical join, such that $x \in \Delta_{x}$ and $y \in \Delta_{y}$.

(b) $\operatorname{Lk} C_{v} \subseteq \Delta^{=}(x)$ for any chamber $C$ of St $y$ and any vertex $v$ of the simplex carrying $y$.

Proof The implication (a) $\Rightarrow$ (b) is clear. Suppose (b) holds. Let $\Sigma$ be an apartment containing $x$ and $y$. Then (b) means that $x$ is contained in any wall bordering $\operatorname{St}_{\Sigma} y$. Therefore $x$ is a point of $\overline{\operatorname{St}_{\Sigma} y}$. Since $d(x, v)=\pi / 2$ for any vertex $v$ of the simplex carrying $y$, we get $d(x, y)=\pi / 2$ from Corollary 1.14 , Lemma 1.11 and the spherical law of cosines. Hence, (a) follows by Proposition 1.15. 

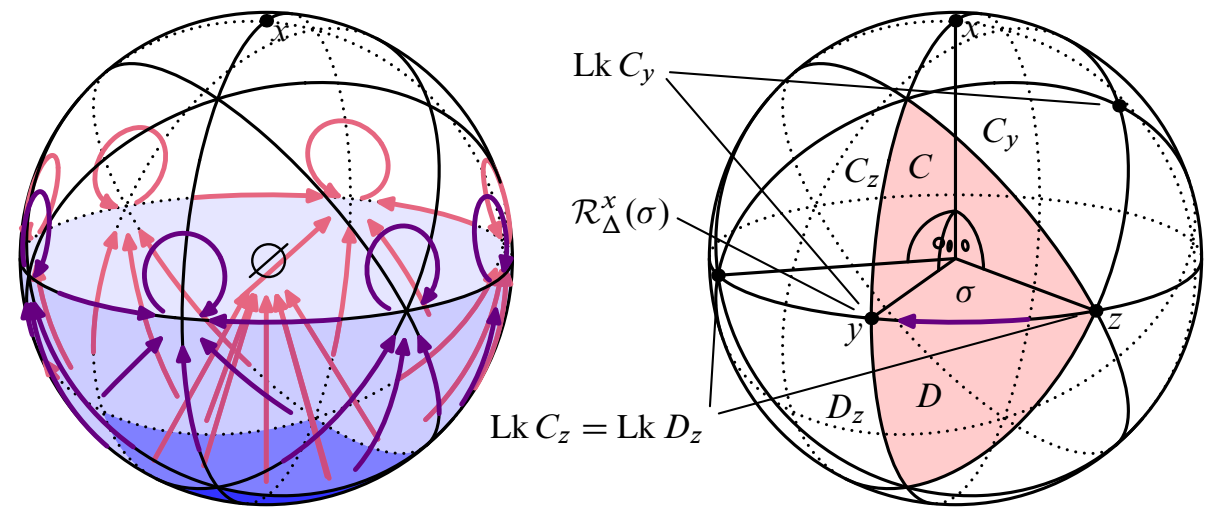

Figure 4: The $(\Delta, x)$-restriction in an apartment of $\operatorname{Flag}\left(\mathbb{P}_{3}(F)\right)$ containing $x$. (Here $x$ is the midpoint of an edge joining a point and a hyperplane of $\mathbb{P}_{3}(F)$.)

Corollary 4.4 Let $\sigma$ be a simplex of $\Delta^{\geq}(x)$. Then

$$
\mathcal{R}_{\Delta}^{x}(\sigma)=\varnothing \Longleftrightarrow \sigma_{x}^{=} \text {is a simplex of } \Delta_{\text {hor }}(x) .
$$

Notation 4.5 For $\sigma \in \mathcal{S}(\Delta)$ let $\lambda_{\sigma}$ denote the simplicial map $\overline{\operatorname{St} \sigma} \rightarrow \operatorname{St} \sigma, \tau \mapsto \sigma \cup \tau$.

Lemma 4.6 Let $\tau$ be a simplex of $\Delta^{=}(x)$ and let $\sigma$ be a face of $\tau$. Then we have $\mathcal{R}_{\mathrm{Lk} \sigma}^{p_{\sigma} x}(\tau \backslash \sigma)=\mathcal{R}_{\Delta}^{x}(\tau) \backslash \sigma$ and $\mathcal{R}_{\Delta}^{x}(\tau) \cap \sigma$ is a face of $\mathcal{R}_{\Delta}^{x}(\sigma)$.

Proof The map $\lambda_{\sigma}$ identifies $\operatorname{St}_{\mathrm{Lk} \sigma}(\tau \backslash \sigma)$ with $\mathrm{St} \tau$. Note that for any vertex $v \in \operatorname{vt}(\tau \backslash \sigma)$ and any chamber $C$ of $\operatorname{St}_{\operatorname{Lk} \sigma}(\tau \backslash \sigma)$ we have $\operatorname{Lk}_{\operatorname{Lk} \sigma} C_{v}=\operatorname{Lk}\left(\lambda_{\sigma} C\right)_{v}$. By Lemma and Definition 3.6 we know that $(\operatorname{Lk} \sigma)=\left(p_{\sigma} x\right)$ equals $\operatorname{Lk} \sigma \cap \Delta^{=}(x)$. Hence, using the definition one gets $\mathcal{R}_{\operatorname{Lk} \sigma}^{p_{\sigma} x}(\tau \backslash \sigma)=\mathcal{R}_{\Delta}^{x}(\tau) \backslash \sigma$. The second assertion is clear, since St $\sigma$ contains St $\tau$.

Corollary 4.7 The restriction map $\mathcal{R}_{\Delta}^{x}$ is idempotent and its image im $\mathcal{R}_{\Delta}^{x}$ is a subcomplex of $\Delta_{\mathrm{ver}}(x)$.

Proof By Lemma 4.6 any face $\sigma$ of $\mathcal{R}_{\Delta}^{x}(\tau)$ is a face of $\mathcal{R}_{\Delta}^{x}(\sigma)$, hence $\mathcal{R}_{\Delta}^{x}(\sigma)=\sigma$ for all faces of $\mathcal{R}_{\Delta}^{x}(\tau)$. According to Corollary 4.4 a simplex $\sigma \in \operatorname{im} \mathcal{R}_{\Delta}^{x}$ is contained $\Delta_{\text {hor }}(x)$ if and only if $\sigma=\mathcal{R}_{\Delta}^{x}(\sigma)=\varnothing$.

Lemma 4.8 Let $\tau$ be a simplex of $\Delta^{\geq}(x)$ and let $\sigma$ be a face of $\tau_{x}^{=}$. The following statements are equivalent:

(a) $(\tau \backslash \sigma)_{x}^{\bar{z}}$ is a simplex of $(\operatorname{Lk} \sigma)_{\mathrm{hor}}\left(p_{\sigma} x\right)$. 

(b) $\mathcal{R}_{\Delta}^{x}(\tau)$ is a face of $\sigma$.
(c) $\mathcal{R}_{\Delta}^{x}(\tau)=\mathcal{R}_{\Delta}^{x}(\sigma)$.

Proof We may suppose $\tau=\tau_{\bar{x}}^{=}$. By Lemma 4.6 and Corollary 4.4 the simplex $\tau \backslash \sigma$ is contained in $(\operatorname{Lk} \sigma)_{\text {hor }}\left(p_{\sigma} x\right)$, if and only if $\mathcal{R}_{\Delta}^{x}(\tau) \backslash \sigma=\varnothing$ holds, hence, if and only if $\mathcal{R}_{\Delta}^{x}(\tau)$ is a face of $\sigma$. Thus, (a) $\Leftrightarrow$ (b).

The implication (c) $\Rightarrow$ (b) is obvious, since $\mathcal{R}_{\Delta}^{x}(\sigma)$ is a face of $\sigma$. We show (b) $\Rightarrow$ (c): Assume $\mathcal{R}_{\Delta}^{x}(\tau)$ is a face of $\sigma$. Then $\mathcal{R}_{\Delta}^{x}(\tau)$ is a face of $\mathcal{R}_{\Delta}^{x}(\sigma)$ according to Lemma 4.6. Further $\tau \backslash \mathcal{R}_{\Delta}^{x}(\tau)$, hence its face $\mathcal{R}_{\Delta}^{x}(\sigma) \backslash \mathcal{R}_{\Delta}^{x}(\tau)$ is contained in $\left(\operatorname{Lk} \mathcal{R}_{\Delta}^{x}(\tau)\right)_{\text {hor }}\left(p_{\mathcal{R}_{\Delta}^{x}(\tau)} x\right)$ by (b) $\Rightarrow$ (a). It follows $\mathcal{R}_{\Delta}^{x}(\sigma) \backslash \mathcal{R}_{\Delta}^{x}(\tau)=\varnothing$ by Corollary 4.4, Lemma 4.6 and Corollary 4.7. So $\mathcal{R}_{\Delta}^{x}(\sigma)=\mathcal{R}_{\Delta}^{x}(\tau)$.

By Lemma 4.8 the $\mathcal{R}_{\Delta}^{x}$-preimage of $\sigma \in \mathcal{S}\left(\mathrm{im} \mathcal{R}_{\Delta}^{x}\right)$ is $\mathrm{St}_{F} \sigma$ for some subcomplex $F \subseteq \Delta^{\geq}(x)$ and $\operatorname{Lk}_{F} \sigma=(\operatorname{Lk} \sigma)^{>}\left(p_{\sigma} x\right) *(\operatorname{Lk} \sigma)_{\text {hor }}\left(p_{\sigma} x\right)$. Moreover: If $\tau \cup \sigma$ is an equatorial simplex of $\mathrm{St}_{F} \sigma$ and $\sigma \backslash \tau$ is contained in $(\operatorname{Lk} \tau)_{\text {hor }}\left(p_{\tau} x\right)$, then $\sigma$ is a face of $\tau$, since Lemma 4.8 implies that $\mathcal{R}_{\Delta}^{x}(\tau)=\mathcal{R}_{\Delta}^{x}(\tau \cup \sigma)=\sigma$. Thus, a filtration whose relative stars are the $\mathcal{R}_{\Delta}^{x}$-preimages of the simplices in $\operatorname{im} \mathcal{R}_{\Delta}^{x}$ will satisfy Proposition 4.1(b).

It also would have been possible to use the equivalence of Lemma 4.8 as a definition of the restriction map. This was done by Bux, Köhl (né Gramlich), Witzel and Wortman $[14 ; 15 ; 16 ; 29]$ to define a filtration of Euclidean buildings whose relative links should get the above properties.

Now we are going to construct a grading on the image of the restriction map. Note that $\operatorname{im} \mathcal{R}_{\Delta}^{x}$ is in general not a full subcomplex of $\Delta_{\text {ver }}(x)$.

Definition 4.9 For two simplices $\sigma, \tau$ of $\operatorname{im} \mathcal{R}_{\Delta}^{x}$ we define $\sigma \preceq \tau$ if and only if the upper bound $\sigma \cup \tau$ exists and $\mathcal{R}_{\Delta}^{x}(\sigma \cup \tau)=\tau$. We write $\sigma \prec \tau$ if and only if $\sigma \preceq \tau$ but not $\tau \preceq \sigma$.

Lemma 4.10 Let $\sigma$ be a simplex of $\Delta^{=}(x)$ and let $\tau$ be a simplex of im $\mathcal{R}_{\Delta}^{x}$. Suppose $\mathcal{R}_{\Delta}^{x}(\sigma) \preceq \tau$. Then $\sigma \cup \tau$ exists and $\mathcal{R}_{\Delta}^{x}(\sigma \cup \tau)=\tau$.

Proof Set $\theta=\mathcal{R}_{\Delta}^{x}(\sigma)$. According to Lemma $4.8 \sigma \backslash \theta$ is a simplex of $(\operatorname{Lk} \theta)_{\text {hor }}\left(p_{\theta} x\right)$, whereas $\tau \backslash \theta=\mathcal{R}_{\Delta}^{x}(\tau \cup \theta) \backslash \theta=\mathcal{R}_{\operatorname{Lk} \theta}^{p_{\theta} x}(\tau \backslash \theta)$ is contained in $(\operatorname{Lk} \theta)_{\text {ver }}\left(p_{\theta} x\right)$ by Corollary 4.7. Hence, $\sigma \cup \tau$ exists.

$\mathcal{R}_{\Delta}^{x}(\sigma \cup \tau) \cap \sigma$ is a face of $\theta$ by Lemma 4.6. Since $\sigma \backslash \theta$ and $\mathcal{R}_{\Delta}^{x}(\sigma \cup \tau)$ are disjoint, the latter is a face of $\theta \cup \tau$. From Lemma 4.8 we get $\mathcal{R}_{\Delta}^{x}(\sigma \cup \tau)=\mathcal{R}_{\Delta}^{x}(\theta \cup \tau)=\tau$. 
Corollary 4.11 $\left(\operatorname{im} \mathcal{R}_{\Delta}^{x}, \preceq\right)$ is a poset with unique minimal element $\varnothing$.

Proof Let $\sigma, \tau$ and $\theta$ be simplices of $\operatorname{im} \mathcal{R}_{\Delta}^{x}$. Suppose $\sigma \preceq \tau$ and $\tau \preceq \theta$. Then the upper bound $\sigma \cup \tau \cup \theta$ exists and $\mathcal{R}_{\Delta}^{x}(\sigma \cup \tau \cup \theta)=\theta$ by Lemma 4.10. From Lemma 4.8 follows $\mathcal{R}_{\Delta}^{x}(\sigma \cup \theta)=\theta$, since $\mathcal{R}_{\Delta}^{x}(\sigma \cup \tau \cup \theta)$ is a face of $\sigma \cup \theta$. Hence, $\sigma \preceq \theta$.

Suppose $\sigma_{0} \prec \sigma_{1} \prec \cdots$ is a strictly increasing sequence in (im $\mathcal{R}_{\Delta}^{x}, \preceq$ ). According to Lemma 4.10 the upper bounds $\sigma_{0} \cup \sigma_{1} \cup \cdots \cup \sigma_{k}$ exist and the sequence of their ranks is strictly increasing. So, the length of any strictly increasing sequence in (im $\mathcal{R}_{\Delta}^{x}, \preceq$ ) is bounded from above by $\mathrm{rk} \Delta_{\mathrm{ver}}^{=}(x)$. That is, strictly increasing sequences are finite.

Definition 4.12 For a simplex $\sigma$ of $\Delta^{\geq}(x)$ let its height ht $(\sigma)$ be the length of the longest strictly increasing chain in (im $\mathcal{R}_{\Delta}^{x}, \preceq$ ) ending with $\mathcal{R}_{\Delta}^{x}(\sigma)$.

Lemma 4.13 Let $\tau$ be a simplex of $\Delta^{\geq}(x)$. Then $h t(\sigma) \leq \mathrm{ht}(\tau)$, for any face $\sigma$ of $\tau$. Equality holds if and only if $\mathcal{R}_{\Delta}^{x}(\sigma)=\mathcal{R}_{\Delta}^{x}(\tau)$.

Proof According to Lemma 4.8 we have $\mathcal{R}_{\Delta}^{x}\left(\mathcal{R}_{\Delta}^{x}(\sigma) \cup \mathcal{R}_{\Delta}^{x}(\tau)\right)=\mathcal{R}_{\Delta}^{x}(\tau)$, for any face $\sigma$ of $\tau$ (replace $\sigma$ by $\mathcal{R}_{\Delta}^{x}(\sigma) \cup \mathcal{R}_{\Delta}^{x}(\tau)$ and use (b) $\Rightarrow\left(\right.$ c)), hence $\mathcal{R}_{\Delta}^{x}(\sigma) \preceq \mathcal{R}_{\Delta}^{x}(\tau)$ and consequently ht $(\sigma) \leq \mathrm{ht}(\tau)$. By definition, we have either $\mathcal{R}_{\Delta}^{x}(\sigma)=\mathcal{R}_{\Delta}^{x}(\tau)$ or $\mathcal{R}_{\Delta}^{x}(\sigma) \prec \mathcal{R}_{\Delta}^{x}(\tau)$ (which means ht $(\sigma)<\operatorname{ht}(\tau)$ ).

We conclude this section by the following corollary that is a more precise version of Proposition 4.1.

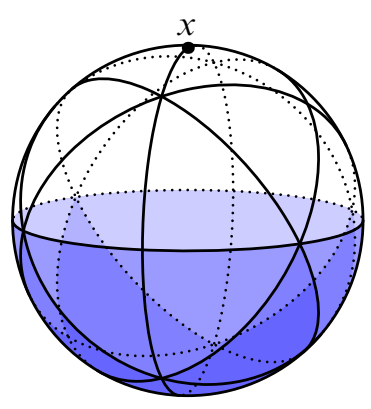

$\mathrm{F}_{2}$

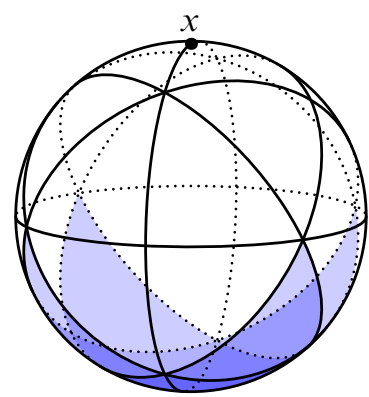

$F_{1}$

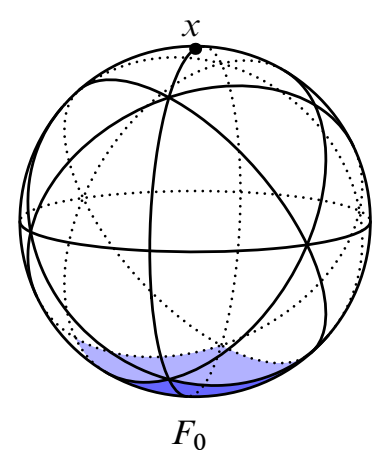

$F_{0}$

Figure 5: The filtration of an apartment of $\operatorname{Flag}\left(\mathbb{P}_{3}(F)\right)$ containing $x$. (Here $x$ is the midpoint of an edge joining a point and a hyperplane of $\mathbb{P}_{3}(F)$.) 
Corollary and Definition 4.14 For $0 \leq k \leq \mathrm{rk} \Delta_{\text {ver }}^{=}(x)$ we define

$$
F_{k}=\bigcup\left\{\sigma \in \mathcal{S}\left(\Delta^{\geq}(x)\right) \mid \mathrm{ht}(\sigma) \leq k\right\} .
$$

Then $\Delta^{>}(x) * \Delta_{\text {hor }}(x)=F_{0} \subseteq F_{1} \subseteq \cdots \subseteq F_{\text {rk } \Delta_{\text {ver }}^{=}(x)}=\Delta^{\geq}(x)$ is sequence of subcomplexes. The complex $F_{k}$ is the disjoint union of the previous complex and the relative stars (in $F_{k}$ ) of the height-k-simplices from im $\mathcal{R}_{\Delta}^{x}$. For any height-k-simplex $\sigma$ from im $\mathcal{R}_{\Delta}^{x}$ the relative star $\mathrm{St}_{F_{k}} \sigma$ is the preimage of $\sigma$ under $(\Delta, x)$-restriction and $\operatorname{Lk}_{F_{k}} \sigma=(\operatorname{Lk} \sigma)^{>}\left(p_{\sigma} x\right) *(\operatorname{Lk} \sigma)_{\text {hor }}\left(p_{\sigma} x\right)$.

\section{Spherical unions of cones}

If, for some simplex $\sigma$, we would like to contract a (dim $\Delta-1)$-spherical subcomplex $L$ of $\partial$ St $\sigma$ without using the points of St $\sigma$, we recognize $L$ as the base of a geodesic cone with tip in some simplex opposite to $\sigma$. But sometimes, we only have cones over some $L$ covering family of subcomplexes at our disposal. In the present section we establish a lemma on $\operatorname{dim} \Delta$-sphericity of a union of cones over subcomplexes of $\partial \mathrm{St} \sigma$, in order to deal with this case.

Notation 5.1 For any pair $\sigma$ op $\tau$ of opposite simplices from $\Delta$ and any simplex $\theta$ of $\overline{\mathrm{St} \sigma}$ we put

$$
\begin{aligned}
\mathcal{C}_{*}^{*}(\sigma, \theta, \tau) & =\operatorname{Conv}\left(\lambda_{\sigma} \theta, \operatorname{proj}_{\tau} \lambda_{\sigma} \theta\right), \\
\mathcal{C}^{*}(\sigma, \theta, \tau) & =\mathcal{C}_{*}^{*}(\sigma, \theta, \tau) \backslash \operatorname{St} \sigma, \\
\mathcal{C}(\sigma, \theta, \tau) & =\mathcal{C}_{*}^{*}(\sigma, \theta, \tau) \backslash(\operatorname{St} \sigma \cup \operatorname{St} \tau) .
\end{aligned}
$$

Recall that $\lambda_{\sigma}$ denotes the simplicial map $\overline{\mathrm{St} \sigma} \rightarrow \operatorname{St} \sigma ; \theta \mapsto \sigma \cup \theta$.

Lemma 5.2 Let $\sigma$ and $\tau$ be opposite simplices of $\Delta$ and let $\theta$ be a simplex of $\overline{\mathrm{St} \sigma}$. Suppose $\mathcal{C}$ is one of the complexes $\mathcal{C}_{*}^{*}(\sigma, \theta, \tau), \mathcal{C}^{*}(\sigma, \theta, \tau)$, or $\mathcal{C}(\sigma, \theta, \tau)$. Then $\mathcal{C} \cap \partial \operatorname{St} \sigma=\partial \lambda_{\sigma} \theta \backslash \operatorname{St} \sigma$.

Proof Any face of $\lambda_{\sigma} \theta$ that is not contained in St $\sigma$ lies in $\mathcal{C}$. Conversely, let $\eta$ be a simplex of $\operatorname{Conv}\left(\lambda_{\sigma} \theta, \operatorname{proj}_{\tau} \lambda_{\sigma} \theta\right) \cap \partial \operatorname{St} \sigma$. Since $\sigma$ is opposite to $\tau$, we have $\lambda_{\sigma} \theta=\operatorname{proj}_{\sigma} \operatorname{proj}_{\tau} \lambda_{\sigma} \theta$. Then $\operatorname{Conv}\left(\lambda_{\sigma} \theta, \operatorname{proj}_{\tau} \lambda_{\sigma} \theta\right)$ equals $\operatorname{Conv}\left(\sigma, \operatorname{proj}_{\tau} \lambda_{\sigma} \theta\right)$ and $\lambda_{\sigma} \eta$ is a face of $\lambda_{\sigma} \theta$, because $\operatorname{proj}_{\sigma} \operatorname{proj}_{\tau} \lambda_{\sigma} \theta$ is the unique maximal simplex of $\operatorname{Conv}\left(\sigma, \operatorname{proj}_{\tau} \lambda_{\sigma} \theta\right) \cap \operatorname{St} \sigma$. Hence, $\eta$ is contained in $\partial \lambda_{\sigma} \theta \backslash \mathrm{St} \sigma$.

Note, that for any point $z$ of $\tau$, the complex $\mathcal{C}^{*}(\sigma, \theta, \tau)$ is the geodesic cone over $\partial \lambda_{\sigma} \theta \backslash$ St $\sigma$ with tip in $z$. 
Notation 5.3 Let $\sigma$ and $\tau$ be opposite simplices of $\Delta$. For any non-empty subcomplex $L$ of $\overline{\mathrm{St} \sigma}$ we put

$$
\mathcal{C}_{*}^{*}(\sigma, L, \tau)=\bigcup_{\theta \in \mathcal{S}(L)} \mathcal{C}_{*}^{*}(\sigma, \theta, \tau)
$$

and analogously $\mathcal{C}^{*}(\sigma, L, \tau)$ as well as $\mathcal{C}(\sigma, L, \tau)$.

Definition 5.4 A subcomplex $\Lambda \subseteq \Delta$ contained in $\Delta \backslash \operatorname{Ant}^{*}(y)$ is quasi-star-shaped with respect to $y$ if and only if for any point $z \in \Lambda$ the segment $\left[z, p_{y} z\right]$ joining $z$ and its geodesic projection on $\partial \mathrm{St} y$ is contained in $\Lambda$. We denote the set of subcomplexes that are quasi-star-shaped with respect to $y$ by $\mathcal{Q}_{y}$.

Observation 5.5 $\mathcal{Q}_{y}$ is closed under unions and intersections.

Observation 5.6 For any $\Lambda \in \mathcal{Q}_{y}$, there is a strong deformation retraction of $\Lambda$ onto $\Lambda \cap \partial$ St $y$ induced by the geodesic projection to $\partial \mathrm{St} y$. In particular $\Lambda$ and $\Lambda \cap \partial \mathrm{St} y$ are homotopy equivalent.

Lemma 5.7 Let $\sigma$ op $\tau$ be opposite simplices of $\Delta$ and let $y$ be a point of $\sigma$. Furthermore let $\theta$ be a simplex of $\overline{\operatorname{St} \sigma}$. Then $\mathcal{C}(\sigma, \theta, \tau)$ is contained in $\mathcal{Q}_{y}$.

Proof Since $\mathcal{C}_{*}^{*}(\sigma, \theta, \tau)$ is contained in an apartment, there is only one antipode $z$ of $y$ in $\mathcal{C}_{*}^{*}(\sigma, \theta, \tau)$. Certainly $z$ is a point of $\tau$. For any point $u \in \mathcal{C}(\sigma, \theta, \tau)$, the geodesic segment $s$ joining $y, z$ going through $u$ is contained in $\mathcal{C}_{*}^{*}(\sigma, \theta, \tau)$, because $s=[y, u] \cup[u, z]$ and $\mathcal{C}_{*}^{*}(\sigma, \theta, \tau)$ is convex. From Lemma 1.10, it follows that $s \backslash(\mathrm{St} y \cup \mathrm{St} z)=\left[p_{y} u, p_{z} u\right]$. Then $\left[p_{y} u, u\right] \subseteq\left[p_{y} u, p_{z} u\right]$ lies in $\mathcal{C}(\sigma, \theta, \tau)$.

Corollary 5.8 Let $\sigma$ and $\tau$ be opposite simplices of $\Delta$ and let $L$ be a subcomplex of $\operatorname{Lk} \sigma$. Then $\mathcal{C}^{*}(\sigma, L, \tau)$ is $(\operatorname{dim} \sigma+\operatorname{dim} L+1)$-dimensional and contractible.

Lemma 5.9 Let $I \neq \varnothing$ be an index set and let $\left\{L_{i} \mid i \in I\right\}$ be a family of non-empty subcomplexes of $\operatorname{Lk} \sigma$. Furthermore let $\left\{\tau_{i} \mid i \in I\right\}$ be a family of simplices opposite to $\sigma$. Suppose $L_{i} \cap \bigcup_{J} L_{j}$ is $\operatorname{dim} \operatorname{Lk} \sigma$-spherical, for any $i \in I$ and any non-empty, finite $J \subseteq I$. Then $\bigcup_{i \in I} \mathcal{C}^{*}\left(\sigma, L_{i}, \tau_{i}\right)$ is $\operatorname{dim} \Delta$-spherical.

Proof For subsets $J \subseteq I$ we put

$$
\mathcal{C}^{*}(J):=\bigcup_{j \in J} \mathcal{C}^{*}\left(\sigma, L_{j}, \tau_{j}\right) \quad \text { and } \quad \mathcal{C}(J):=\bigcup_{j \in J} \mathcal{C}\left(\sigma, L_{j}, \tau_{j}\right)
$$

Since it is sufficient to give a proof for $\# I<\infty$, we use induction on $\# I$. The case $\# I=1$ is clear by Corollary 5.8. 
Now assume $\# I>1$. We put $J=I \backslash\{i\}$ and $J^{\prime}=\left\{j \in J \mid \tau_{j}=\tau_{i}\right\}$ for some $i \in I$. Let $y$ be a point of $\sigma$ and let $\alpha: \operatorname{Lk} \sigma \rightarrow \operatorname{Lk} \tau_{i}$ denote the isomorphism induced by $\operatorname{proj}_{\tau_{i}}$. Recall that there is a labeling on $\Delta$. Since all $\tau_{j}$ have the same labels, St $\tau_{j} \cap \mathrm{St} \tau_{l}$ is empty, unless $\tau_{j}=\tau_{l}$. Furthermore $\operatorname{St} \tau_{i} \cap \mathcal{C}(J)$ and $\operatorname{St} \tau_{i} \cap \mathcal{C}(\{i\})$ are empty. We therefore get

$$
\begin{aligned}
\mathcal{C}^{*}(\{i\}) \cap \mathcal{C}^{*}(J) & =\left(\mathcal{C}(\{i\}) \cup \overline{\operatorname{St}_{\mathcal{C}^{*}(\{i\})} \tau_{i}}\right) \cap\left(\mathcal{C}(J) \cup \overline{\operatorname{St}_{\mathcal{C}^{*}\left(J^{\prime}\right)} \tau_{i}}\right) \\
& =(\mathcal{C}(\{i\}) \cap \mathcal{C}(J)) \cup\left(\overline{\operatorname{St}_{\mathcal{C}^{*}(\{i\})} \tau_{i}} \cap \overline{\operatorname{St}_{\mathcal{C}^{*}\left(J^{\prime}\right)} \tau_{i}}\right) \\
& =(\mathcal{C}(\{i\}) \cap \mathcal{C}(J)) \cup \overline{\lambda_{\tau_{i}}\left(\alpha L_{i} \cap \bigcup_{j \in J^{\prime}} \alpha L_{j}\right)}
\end{aligned}
$$

The second complex of this union is contractible, since it is a cone with tip in $\tau_{i}$. According to Lemma 5.7 and Observation 5.5, the first complex is contained in $\mathcal{Q}_{y}$; and by Observation 5.6 and Lemma 5.2, it is homotopy equivalent to the (dim $\Delta-1)-$ spherical complex

$$
\begin{aligned}
\partial \operatorname{St} \sigma \cap \mathcal{C}(\{i\}) \cap \mathcal{C}(J) & =\left(\partial \operatorname{St} \sigma \cap \mathcal{C}\left(\sigma, L_{i}, \tau_{i}\right)\right) \cap \bigcup_{j \in J}\left(\partial \operatorname{St} \sigma \cap \mathcal{C}\left(\sigma, L_{j}, \tau_{j}\right)\right) \\
& =\left(\partial \sigma * L_{i}\right) \cap \bigcup_{j \in J}\left(\partial \sigma * L_{j}\right)=\partial \sigma *\left(L_{i} \cap \bigcup_{j \in J} L_{j}\right)
\end{aligned}
$$

Their intersection

$$
(\mathcal{C}(\{i\}) \cap \mathcal{C}(J)) \cap \overline{\lambda_{\tau_{i}}\left(\alpha L_{i} \cap \bigcup_{j \in J^{\prime}} \alpha L_{j}\right)}=\left(\alpha L_{i} \cap \bigcup_{j \in J^{\prime}} \alpha L_{j}\right) * \partial \tau_{i}
$$

is $(\operatorname{dim} \Delta-1)$-spherical as well. Then $\mathcal{C}^{*}(\{i\}) \cap \mathcal{C}^{*}(J)$ is $(\operatorname{dim} \Delta-1)$-spherical by Lemma $1.1\left(\right.$ a). Hence, $\mathcal{C}^{*}(I)$ is $\operatorname{dim} \Delta$-spherical by the induction hypothesis, Corollary 5.8 and again by Lemma 1.1(a).

\section{Some subcomplexes of $F_{k}$}

We intent to deduce the $\operatorname{dim} \Delta$-sphericity of a filtration stage $F_{k-1}$ from those of the next stage $F_{k}$ using Lemma 1.1 (b) in order to proof the $\operatorname{dim} \Delta$-sphericity of $F_{0}=$ $\Delta^{>}(x)$. Therefore we need a dim $\Delta$-spherical subcomplex of $F_{\mathrm{ht}(\sigma)-1}$ containing the boundary of the relative $\operatorname{star} \operatorname{St}_{F_{\mathrm{ht}(\sigma)}} \sigma$ for any simplex $\sigma \neq \varnothing$ of $\operatorname{im} \mathcal{R}_{\Delta}^{x}$. The cones $\mathcal{C}^{*}\left(\sigma, \partial \operatorname{St}_{F_{\mathrm{ht}(\sigma)}} \sigma, \tau\right)$ for some opposite $\tau$ of $\sigma$ would be good candidates. But unfortunately, an opposite $\tau$ of $\sigma$ such that the cone over $\partial \mathrm{St}_{F_{\mathrm{ht}(\sigma)}} \sigma$ with tip in $\tau$ is contained in $F_{\mathrm{ht}(\sigma)-1}$ may not exist. In order to deal with this obstruction, we already proofed Lemma 5.9 to establish a criterion on $\operatorname{dim} \Delta$-sphericity of a union of cones over subcomplexes of $\partial \mathrm{St} \sigma$. In this section we construct subcomplexes $L$ of $\partial \mathrm{St}_{\mathrm{ht}_{(\sigma)}} \sigma$ and corresponding opposites $\tau$ such that $\mathcal{C}^{*}(\sigma, L, \tau)$ is contained in $F_{\mathrm{ht}(\sigma)-1}$. The first step is to reduce the question, whether $\mathcal{C}^{*}(\sigma, L, \tau)$ is contained in 
$F_{\text {ht }(\sigma)-1}$ to the question, whether there is a simplex $\theta$ of $L \operatorname{such}$ that $\operatorname{proj}_{\theta} \tau$ is an equatorial simplex.

Lemma 6.1 Let $\sigma$ be a simplex of $\Delta^{=}(x)$ and let $\tau$ be a simplex opposite to $\sigma$. Then $\mathcal{C}_{*}^{*}(\sigma, L, \tau)$ is a subcomplex of $\Delta^{\geq}(x)$ for any subcomplex $L$ of $\partial \mathrm{St}_{\Delta^{\geq}(x)} \sigma$.

Proof Let $y \in \sigma$ be a point and let $z$ be its antipode in $\tau$. From the triangle inequality we get $d(z, x) \geq \pi / 2$. Let $u$ be a point of $\mathcal{C}_{*}^{*}(\sigma, L, \tau) \backslash\{y, z\}$. Then $u \in \mathcal{C}_{*}^{*}(\sigma, \theta, \tau)$ for some simplex $\theta \in \mathcal{S}(L)$. The projection $p_{y} u$ of $u$ on $\partial \operatorname{St} \sigma$ is a point of $\partial \lambda_{\sigma} \theta$ by Lemma 5.2. It holds $d\left(p_{y} u, x\right) \geq \pi / 2$, because $\lambda_{\sigma} \theta$ is a simplex of $\Delta^{\geq}(x)$. Hence, $\angle_{y}(x, u)$ is not acute and we obtain $d(x, u) \geq \pi / 2$ by the spherical law of cosines.

Lemma 6.2 Let $\sigma \neq \varnothing$ be a simplex of im $\mathcal{R}_{\Delta}^{x}$ and let $\tau$ be opposite to $\sigma$. Let $L$ be a subcomplex of $\partial \mathrm{St}_{F_{\mathrm{ht}(\sigma)}} \sigma$. If $\mathcal{C}_{*}^{*}(\sigma, L, \tau) \cap \Delta^{=}(x)$ is contained in $\overline{\mathrm{St}} \sigma$, then $\mathcal{C}_{*}^{*}(\sigma, L, \tau)$ is a subcomplex of $F_{\mathrm{ht}(\sigma)}$ and $\mathcal{C}^{*}(\sigma, L, \tau)=\mathcal{C}_{*}^{*}(\sigma, L, \tau) \cap F_{\mathrm{ht}(\sigma)-1}$.

Proof Let $\theta$ be a simplex of $\mathcal{C}^{*}(\sigma, L, \tau)$. Then $\theta \in \mathcal{S}\left(\Delta^{\geq}(x)\right)$ by Lemma 6.1. Either $\theta$ is a simplex of $\Delta^{>}(x)$, which means $h t(\theta)=0<\operatorname{ht}(\sigma)$, or we have $\theta_{x}^{=} \neq \varnothing$ (see Definition 4.2). In the latter case $\theta_{x}^{=}$is contained in $\partial \lambda_{\sigma} \eta \backslash \operatorname{St} \sigma$ for some simplex $\eta \in \mathcal{S}(L)$ by Lemma 5.2. Since $\sigma$ is not a face of $\theta_{x}^{=}$, we get $\mathcal{R}_{\Delta}^{x}\left(\theta_{x}^{=}\right) \neq \mathcal{R}_{\Delta}^{x}\left(\lambda_{\sigma} \eta\right)=\sigma$ from Lemma 4.8. Then $\operatorname{ht}(\theta)=\operatorname{ht}\left(\theta_{x}^{=}\right)<\operatorname{ht}\left(\lambda_{\sigma} \eta\right)=\mathrm{ht}(\sigma)$ according to Lemma 4.13. Therefore $\mathcal{C}^{*}(\sigma, L, \tau)$ is a subcomplex of $F_{\mathrm{ht}(\sigma)-1}$. The claim follows, since any simplex of $\mathcal{C}_{*}^{*}(\sigma, L, \tau)$ is either a simplex of $\mathcal{C}^{*}(\sigma, L, \tau)$ or lies in $\operatorname{St}_{F_{\mathrm{ht}(\sigma)}} \sigma$ and $F_{\mathrm{ht}(\sigma)-1} \subseteq F_{\mathrm{ht}(\sigma)} \backslash \mathrm{St}_{F_{\mathrm{ht}(\sigma)}} \sigma$ by Corollary and Definition 4.14.

Lemma 6.3 Let $\sigma \neq \varnothing$ be a simplex of $\Delta^{=}(x)$ and let $\tau$ be opposite to $\sigma$. Let $L$ be a subcomplex of $\partial \operatorname{St}_{\Delta^{\geq}(x)} \sigma$. If $\mathcal{C}_{*}^{*}(\sigma, L, \tau) \cap \Delta^{=}(x)$ is not contained in $\overline{\mathrm{St}} \sigma$, then $\operatorname{proj}_{\theta} \tau \in \mathcal{S}\left(\Delta^{=}(x)\right)$ for some simplex $\theta$ of $\Delta^{=}(x) \cap\left(\overline{\lambda_{\sigma} L} \backslash\right.$ St $\left.\sigma\right)$.

Proof Recall that we are dealing with open simplices. Therefore, a simplex contained in a closed hemisphere lies entirely in the associated equator if it carries a point of the equator.

Let $u$ be a point of $\Delta^{=}(x) \cap \mathcal{C}_{*}^{*}(\sigma, L, \tau)$ that is not contained in $\overline{\mathrm{St} \sigma}$. Furthermore let $y \in \sigma$ and $z \in \tau$ be antipodal points. If $u=z$, then $\tau=\operatorname{proj}_{\varnothing} \tau$ is a simplex of $\Delta^{=}(x)$ by Lemma 6.1. Hence, suppose $u \neq z$.

The segment $[y, u]$ lies in $\Omega \frac{\leq}{\Delta}(x)$ as well as in $\mathcal{C}_{*}^{*}(\sigma, L, \tau) \subseteq \Omega_{\Delta}^{\geq}(x)$. Therefore $[y, u]$ is entirely contained in $\Omega_{\Delta}^{=}(x)$. By Lemma 5.2 the simplex $\theta$ carrying $p_{y} u$ is in $\overline{\lambda_{\sigma} L} \backslash$ St $\sigma$ and also a simplex of $\Delta^{=}(x)$ by Lemma 6.1. The projection $p_{y} u$ lies on 
the geodesic segment joining $y$ and $z$ going through $u$. We obtain $\left[p_{y} u, u\right] \subset\left[p_{y} u, z\right]$, implying by Lemma 1.10 that $\operatorname{proj}_{\theta} \tau$ carries a point of $\Omega_{\bar{\Delta}}^{\bar{\nu}}(x)$. Then $\operatorname{proj}_{\theta} \tau$ is a simplex of $\Delta^{=}(x)$ according to Lemma 6.1.

Let $\sigma$ be a non-empty simplex of $\operatorname{im} \mathcal{R}_{\Delta}^{x}$. Now, we will show that $\mathcal{C}^{*}(\sigma, L, \tau)$ is contained in $F_{\mathrm{ht}(\sigma)-1}$ for some opposite $\tau$ of $\sigma$, provided $\Delta$ is thick and $L$ is a subcomplex of $\partial \mathrm{St}_{F_{\mathrm{ht}(\sigma)}} \sigma$ such that $L \cap \Delta=(x) \subseteq \Sigma$ for some apartment $\Sigma$ containing $x$ and $\sigma$. We begin by a sketch of the idea:

Let $y$ be a point of $\sigma$. We denote the simplex carrying $x$ by $\xi$ and the simplex carrying $p_{y} x$ by $\chi$. Let $\Sigma$ be an apartment containing $\xi$ and $\sigma$. Further, let $\theta$ be an equatorial simplex of $\Sigma \cap \partial \operatorname{St} \sigma$ and let $\Sigma^{\prime}$ be some other apartment that contains $\chi, \sigma$ and $\theta$. We choose a point $u$ of $\theta$. Denote the opposite of $\sigma$ in $\Sigma^{\prime}$ by $\tau$ and the antipode of $y$ in $\tau$ by $z$.

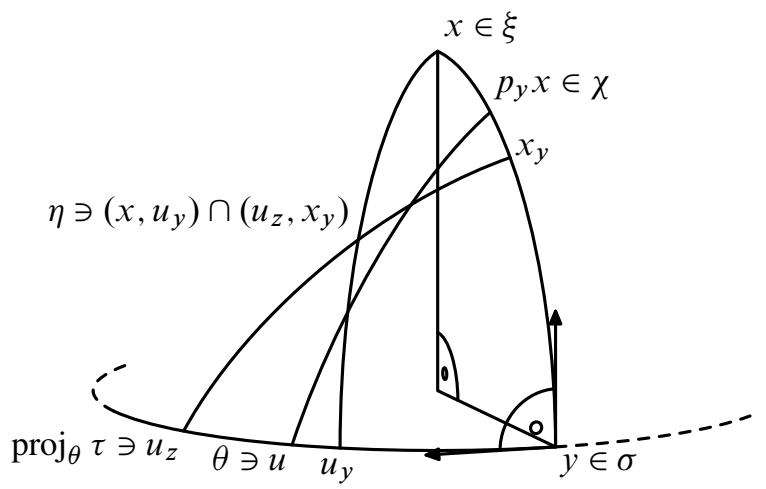

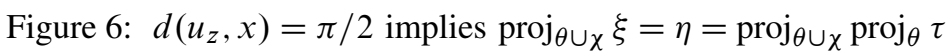

Suppose $\operatorname{proj}_{\theta} \tau$ is an equatorial simplex. Hence $\operatorname{proj}_{\theta} \tau$ contains a point $u_{z} \in[z, u)$ at distance $\pi / 2$ to $x$. By the spherical law of cosines the triangle $\left(u_{z}, y, x\right)$ is contained in some apartment. Then for any $u_{y} \in(u, y)$ and any $x_{y} \in\left(y, p_{y} x\right)$, the segments $\left(x, u_{y}\right),\left(u_{z}, x_{y}\right)$ and $\left(u, p_{y} x\right)$ intersect each other (see Figure 6). We can choose $u_{y}$ and $x_{y}$ such that $\left(x, u_{y}\right) \cap\left(u_{z}, x_{y}\right)$ is near $\left(u, p_{y} x\right)$ but outside the triangle $\left(u, y, p_{y} x\right)$. With this choice the simplex $\eta$ carrying $\left(x, u_{y}\right) \cap\left(u_{z}, x_{y}\right)$ is not contained in $\overline{\mathrm{St} \sigma}$. We obtain $\operatorname{proj}_{\theta \cup \chi} \xi=\eta=\operatorname{proj}_{\theta \cup \chi} \operatorname{proj}_{\theta} \tau=\operatorname{proj}_{\theta \cup \chi} \tau$ by Lemma 1.10. Therefore $\Sigma \cap \Sigma^{\prime}$ is not contained in $\overline{\mathrm{St} \sigma}$.

Thus, $\operatorname{proj}_{\theta} \tau$ is not an equatorial simplex for any opposite $\tau$ of $\sigma$ chosen in an apartment $\Sigma^{\prime}$ satisfying $\Sigma \cap \Sigma^{\prime}=\overline{\mathrm{St}_{\Sigma} \sigma}$. Provided $\Delta$ is thick, such an apartment exists by the following lemma, which follows directly from Abramenko and Van Maldeghem [6, Proposition 4.1]. 
Lemma 6.4 Let $\Delta$ be a thick spherical building. If $K$ is a non-empty, convex chamber subcomplex of an apartment $\Sigma$ of $\Delta$, then there exists an apartment $\Sigma^{\prime}$ of $\Delta$ such that $K=\Sigma \cap \Sigma^{\prime}$.

Lemma 6.5 Let $\sigma \neq \varnothing$ be a simplex of $\operatorname{im} \mathcal{R}_{\Delta}^{x}$ and let $\Sigma, \Sigma^{\prime}$ be apartments such that $x \in \Sigma$ and $\Sigma \cap \Sigma^{\prime}$ contains $\operatorname{St}_{\Sigma} \sigma$. Let $\tau$ be the opposite of $\sigma$ in $\Sigma^{\prime}$. If $\operatorname{proj}_{\theta} \tau$ is contained in $\mathcal{S}\left(\Delta^{=}(x)\right)$ for some simplex $\theta$ of $\Sigma^{=}(x) \cap \partial \operatorname{St}_{F_{\mathrm{ht}(\sigma)}} \sigma$, then $\Sigma \cap \Sigma^{\prime}$ is strictly greater than $\overline{\mathrm{St}_{\Sigma} \sigma}$.

Proof Let $\theta$ be a simplex of $\Sigma^{=}(x) \cap \partial \operatorname{St}_{F_{\mathrm{ht}(\sigma)}} \sigma$ and $\operatorname{proj}_{\theta} \tau \in \mathcal{S}\left(\Delta^{=}(x)\right)$. At first we show that if suffices to consider the case $\theta=\varnothing$ and $\tau \in \mathcal{S}\left(\Delta^{=}(x)\right)$.

We have $\mathcal{R}_{\Delta}^{x}(\sigma \cup \theta)=\sigma$ by Corollary and Definition 4.14, hence $\sigma \backslash \theta$ is a non-empty simplex of $\operatorname{im} \mathcal{R}_{\operatorname{Lk} \theta}^{p_{\theta} x}$ according to Lemma 4.6. The star of $\sigma \backslash \theta$ in $\operatorname{Lk}_{\Sigma} \theta$ is contained in the apartment $\operatorname{Lk}_{\Sigma^{\prime}} \theta$ and $p_{\theta} x$ lies in $\operatorname{Lk}_{\Sigma} \theta$. From Lemma 1.10 we know that

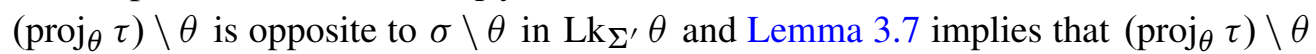
is in the equator complex of $\operatorname{Lk} \theta$ with respect to $p_{\theta} x$ if and only if $\operatorname{proj}_{\theta} \tau$ is a simplex of $\Delta^{=}(x)$. If $\operatorname{Lk}_{\Sigma} \theta \cap \operatorname{Lk}_{\Sigma^{\prime}} \theta$ is strictly greater than the closed star of $\sigma \backslash \theta$ in $\operatorname{Lk}_{\Sigma} \theta$, then $\Sigma \cap \Sigma^{\prime}$ is also strictly greater than $\overline{\operatorname{St}_{\Sigma} \sigma}$. Hence, suppose $\theta=\varnothing$ and $\tau \in \mathcal{S}\left(\Delta^{=}(x)\right)$.

Let $y \in \sigma$ and $z \in \tau$ be antipodal points. Denote the simplex carrying $x$ by $\xi$ and the simplex carrying $p_{y} x$ by $\chi$. Since $d(y, x)+d(x, z)=\pi$, there is a geodesic segment $s$ joining $y$ and $z$ going through $x$. It holds $s \backslash \overline{\operatorname{St} \sigma}=\left[z, p_{y} x\right)$. Since $\mathcal{R}_{\Delta}^{x}(\sigma)=\sigma \neq \varnothing$, the pole $x$ can not be a point of $\overline{\mathrm{St} \sigma}$ according to Proposition 1.15 and Corollary 4.4. Then $x$ is an interior point of $\left[z, p_{y} x\right]$. By Lemma 1.10, it follows that $\operatorname{proj}_{\chi} \xi$ is not contained in $\overline{\operatorname{St} \sigma}$ and also that $\operatorname{proj}_{\chi} \xi=\operatorname{proj}_{\chi} \tau$ is a simplex of $\Sigma \cap \Sigma^{\prime}$.

Corollary 6.6 Let $\Delta$ be thick and let $\sigma \neq \varnothing$ be simplex of $\operatorname{im} \mathcal{R}_{\Delta}^{x}$. If $L$ is a subcomplex of $\mathrm{Lk}_{F_{\mathrm{ht}(\sigma)}} \sigma$ such that $L \cap \Delta^{=}(x) \subseteq \Sigma$ for some apartment $\Sigma$ containing $x$ and $\sigma$, then there exists an opposite $\tau$ of $\sigma$ such that $\mathcal{C}_{*}^{*}(\sigma, L, \tau)$ is a subcomplex of $F_{\mathrm{ht}(\sigma)}$ and $\mathcal{C}^{*}(\sigma, L, \tau)=\mathcal{C}_{*}^{*}(\sigma, L, \tau) \cap F_{\mathrm{ht}(\sigma)-1}$.

Proof By Lemma 6.4 there is an apartment $\Sigma^{\prime}$ such that $\Sigma \cap \Sigma^{\prime}=\overline{\operatorname{St}_{\Sigma} \sigma}$. Let $\tau$ be the opposite of $\sigma$ in $\Sigma^{\prime}$. Then for any simplex $\theta$ of $\Sigma^{=}(x) \cap \partial \operatorname{St}_{F_{\mathrm{ht}(\sigma)}} \sigma$, the projection $\operatorname{proj}_{\theta} \tau$ is not a simplex of $\Delta^{=}(x)$ by Lemma 6.5. Since $\Delta^{=}(x) \cap\left(\overline{\lambda_{\sigma} L} \backslash\right.$ St $\left.\sigma\right)$ is contained in $\Sigma^{=}(x) \cap \partial \operatorname{St}_{F_{\mathrm{ht}(\sigma)}} \sigma$, we obtain $\mathcal{C}_{*}^{*}(\sigma, L, \tau) \cap \Delta^{=}(x) \subseteq \overline{\mathrm{St} \sigma}$ by Lemma 6.3. Now, the assertion follows from Lemma 6.2. 


\section{Proof of Proposition 3.8}

Now, we have got all pieces that are needed to complete the proof of Proposition 3.8.

Proposition 7.1 Let $\Delta$ be a thick spherical building. Then $\Delta^{>}(x)$ is $\operatorname{dim} \Delta_{\text {ver }}(x)-$ spherical.

Proof We use induction on $d=\operatorname{dim} \Delta^{=}(x)$. If $\Delta^{=}(x)$ is empty then $\Delta_{\text {ver }}(x)=\Delta$ and $\Delta^{>}(x)=\Delta^{\geq}(x)$ is $\operatorname{dim} \Delta$-spherical by Corollary 3.2. Let $d \geq 0$ and suppose, open hemisphere complexes with equator complex of dimension less than $d$ are spherical.

If $\Delta_{\text {hor }}(x) \neq \varnothing$, we are done by Proposition 3.5 and the induction hypothesis. Hence, assume that $\Delta=\Delta_{\text {ver }}(x)$. We show that for any non-empty simplex $\sigma$ of im $\mathcal{R}_{\Delta}^{x}$ there is a complex $K_{\sigma}$ that satisfies the following two conditions.

Condition 1: $K_{\sigma} \subseteq F_{\mathrm{ht}(\sigma)}$ and $K_{\sigma}=\mathrm{St}_{F_{\mathrm{ht}(\sigma)}} \sigma \cup\left(K_{\sigma} \cap F_{\mathrm{ht}(\sigma)-1}\right)$.

Condition 2: $\quad K_{\sigma} \cap F_{\mathrm{ht}(\sigma)-1}$ is $\operatorname{dim} \Delta$-spherical.

Assuming, we have such $K_{\sigma}$, we argue as follows: For $1 \leq k \leq d+1$, let $I_{k}$ denote the set of simplices from im $\mathcal{R}_{\Delta}^{x}$ at height $k$. Since $\mathrm{St}_{F_{k}} \sigma \cap \mathrm{St}_{F_{k}} \tau=\varnothing$, for $\sigma, \tau \in I_{k}$ with $\sigma \neq \tau$ by Corollary and Definition 4.14, we obtain by the first condition:

$$
F_{k}=F_{k-1} \cup \bigcup_{\sigma \in I_{k}} K_{\sigma} \text { and } K_{\sigma} \cap K_{\tau} \subseteq F_{k-1} \text {, for } \sigma, \tau \in I_{k} \text { with } \sigma \neq \tau \text {. }
$$

Then $F_{k-1}$ is $\operatorname{dim} \Delta$-spherical provided the same holds for $F_{k}$, by Lemma 1.1 (b) and the second condition. Recall that $\Delta_{\text {hor }}(x)$ is empty. Hence, $\Delta^{>}(x)=F_{0}$ is $\operatorname{dim} \Delta$-spherical, since $F_{d+1}=\Delta^{\geq}(x)$ is $\operatorname{dim} \Delta$-spherical by Corollary 3.2.

It remains to find the complexes $K_{\sigma}$. Let $\sigma$ be a non-empty simplex of im $\mathcal{R}_{\Delta}^{x}$. We put $L=(\operatorname{Lk} \sigma)^{>}\left(p_{\sigma} x\right)$. There are two cases:

Case $1(\operatorname{Lk} \sigma)_{\text {hor }}\left(p_{\sigma} x\right)=\varnothing$ In this case we have $\operatorname{Lk}_{F_{\mathrm{ht}(\sigma)}} \sigma=L$ by Corollary and Definition 4.14. Since $L \cap \Delta^{=}(x)$ is empty and $\Delta$ is thick, Corollary 6.6 provides us with an opposite $\tau$ op $\sigma$ such that $\mathcal{C}_{*}^{*}(\sigma, L, \tau)$ is a subcomplex of $F_{\mathrm{ht}(\sigma)}$ and $\mathcal{C}^{*}(\sigma, L, \tau)=\mathcal{C}_{*}^{*}(\sigma, L, \tau) \cap F_{\mathrm{ht}(\sigma)-1}$. Certainly, $\mathcal{C}_{*}^{*}(\sigma, L, \tau)=\operatorname{St}_{F_{\mathrm{ht}(\sigma)}} \sigma \cup \mathcal{C}^{*}(\sigma, L, \tau)$ since $\mathrm{St}_{\mathrm{ht}_{(\sigma)}} \sigma=\lambda_{\sigma} L$. Furthermore $\mathcal{C}^{*}(\sigma, L, \tau)$ is $\operatorname{dim} \Delta$-spherical by Corollary 5.8. Hence, $K_{\sigma}=\mathcal{C}_{*}^{*}(\sigma, L, \tau)$ satisfies the two conditions above.

Case $2(\operatorname{Lk} \sigma)_{\text {hor }}\left(p_{\sigma} x\right) \neq \varnothing$ In this case we further put $L_{h}=(\operatorname{Lk} \sigma)_{\text {hor }}\left(p_{\sigma} x\right)$. Let $C$ be a chamber of $L_{h}$ and let $\mathcal{A}$ denote the set of apartments of $L_{h}$ that contain $C$. 
We show that for any apartment $A \in \mathcal{A}$, there is an apartment $\Sigma_{A}$ of $\Delta$ that contains $x$ and $\overline{\lambda_{\sigma} A}$.

Let $C^{\prime}$ be opposite to $C$ in $A$. We choose points $y \in C \cup \sigma$ and $y^{\prime} \in C^{\prime} \cup \sigma$ and look at the triangle $\left(x, y, y^{\prime}\right)$. Since $d(x, y)=\pi / 2=d\left(x, y^{\prime}\right)$ and $\angle_{y}\left(x, y^{\prime}\right)=\pi / 2$, equality holds in the spherical law of cosines. Hence, there is an apartment $\Sigma_{A}$ that contains $x$ and $\overline{\lambda_{\sigma} A}=\operatorname{Conv}\left(C \cup \sigma, C^{\prime} \cup \sigma\right)$.

Let $A \in \mathcal{A}$ be arbitrary. Since $(L * A) \cap \Delta^{=}(x)$ is contained in $\Sigma_{A}$ and $\Delta$ is thick, we get an opposite $\tau_{A}$ of $\sigma$ by Corollary 6.6 such that $\mathcal{C}_{*}^{*}\left(\sigma, L * A, \tau_{A}\right)$ is a subcomplex of $F_{\mathrm{ht}(\sigma)}$ and $\mathcal{C}^{*}\left(\sigma, L * A, \tau_{A}\right)=\mathcal{C}_{*}^{*}\left(\sigma, L * A, \tau_{A}\right) \cap F_{\mathrm{ht}(\sigma)-1}$. We define

$$
K_{\sigma}=\bigcup_{A \in \mathcal{A}} \mathcal{C}_{*}^{*}\left(\sigma, L * A, \tau_{A}\right) \text { and } K_{\sigma}^{\prime}=\bigcup_{A \in \mathcal{A}} \mathcal{C}^{*}\left(\sigma, L * A, \tau_{A}\right) .
$$

Then $K_{\sigma}$ is a subcomplex of $F_{\mathrm{ht}(\sigma)}$, and $K_{\sigma}^{\prime}=K_{\sigma} \backslash \mathrm{St} \sigma$ is its intersection with $F_{\mathrm{ht}(\sigma)-1}$. From Corollary and Definition 4.14, we know that $\operatorname{Lk}_{F_{\mathrm{ht}(\sigma)}} \sigma=L * L_{h}$. Since $L_{h}$ is covered by $\mathcal{A}$, the link of $\sigma$ in $K_{\sigma}$ is also $L * L_{h}$. Therefore, the stars of $\sigma$ in $K_{\sigma}$ and $F_{\mathrm{ht}(\sigma)}$ coincide. Hence, $K_{\sigma}=\mathrm{St}_{F_{\mathrm{ht}(\sigma)}} \sigma \cup K_{\sigma}^{\prime}$ satisfies the first condition.

The open hemisphere complex $L$ is $\operatorname{dim}(\operatorname{Lk} \sigma)_{\text {ver }}\left(p_{\sigma} x\right)$-spherical by the induction hypothesis, since $\operatorname{dim}(\operatorname{Lk} \sigma)=\left(p_{\sigma} x\right)<d$. For any $A \in \mathcal{A}$ and any non-empty, finite $\mathcal{A}^{\prime} \subseteq \mathcal{A}$, the intersection $A \cap \bigcup \mathcal{A}^{\prime}$ is a union of convex subcomplexes of $A$ each of which contains $C$. Therefore $A \cap \bigcup \mathcal{A}^{\prime}$ equals $A$ or is contractible. Then

$$
(L * A) \cap \bigcup_{A^{\prime} \in \mathcal{A}^{\prime}}\left(L * A^{\prime}\right)=L *\left(A \cap \bigcup \mathcal{A}^{\prime}\right)
$$

is $\operatorname{dim} \operatorname{Lk} \sigma$-spherical. From Lemma 5.9 we now get the $\operatorname{dim} \Delta$-sphericity of the complex $K_{\sigma}^{\prime}=K_{\sigma} \cap F_{\mathrm{ht}(\sigma)-1}$, hence the second condition.

Proposition 7.2 Let $\Delta$ be a thick spherical building. Then $\Delta^{>}(x)$ is non-contractible.

Proof By Proposition 3.5 we suppose $\Delta_{\text {hor }}(x)=\varnothing$. We show the existence of a $\operatorname{dim} \Delta$-sphere in $\Delta^{>}(x)$ by induction on $\operatorname{dim} \Delta$.

If $\operatorname{dim} \Delta=0$, then $\Omega \leq \frac{\leq}{\Delta}(x)=\{x\}$ is a single point. Since $\Delta$ is thick, $\Delta^{>}(x)=\Delta \backslash\{x\}$ contains a $0-$ sphere.

Let $\operatorname{dim} \Delta>0$ and suppose, open hemisphere complexes of dimension less than $\operatorname{dim} \Delta$ contain a top-dimensional sphere. If $\Delta^{=}(x)=\varnothing$, then $\Delta^{>}(x)$ is a closed, coconvex supported subcomplex and the assertion follows from Corollary 3.2. We therefore assume $\Delta^{=}(x) \neq \varnothing$.

Let $y \in F_{1} \backslash F_{0}$ be a vertex. By Corollary and Definition 4.14, its relative link $L=\mathrm{Lk}_{F_{1}} y$ is a subcomplex of $F_{0}=\Delta^{>}(x)$ and an open hemisphere complex 
of $\operatorname{Lk} y$. According to the induction hypothesis, there is a $(\operatorname{dim} \Delta-1)$-dimensional sphere $S \subseteq L$. Suppose $y$ has two opposites $z^{\prime}, z^{\prime \prime} \in \Delta^{>}(x)$. By Lemma 6.2 and Lemma 6.3, the union $\mathcal{C}^{*}\left(y, L, z^{\prime}\right) \cup \mathcal{C}^{*}\left(y, L, z^{\prime \prime}\right)$ is a subcomplex of $\Delta^{>}(x)$. This complex contains the two geodesic cones over $S$ with tip in $z^{\prime}$ and $z^{\prime \prime}$, hence it contains a $\operatorname{dim} \Delta$-sphere. It remains to show that $y$ has two opposites in $\Delta^{>}(x)$.

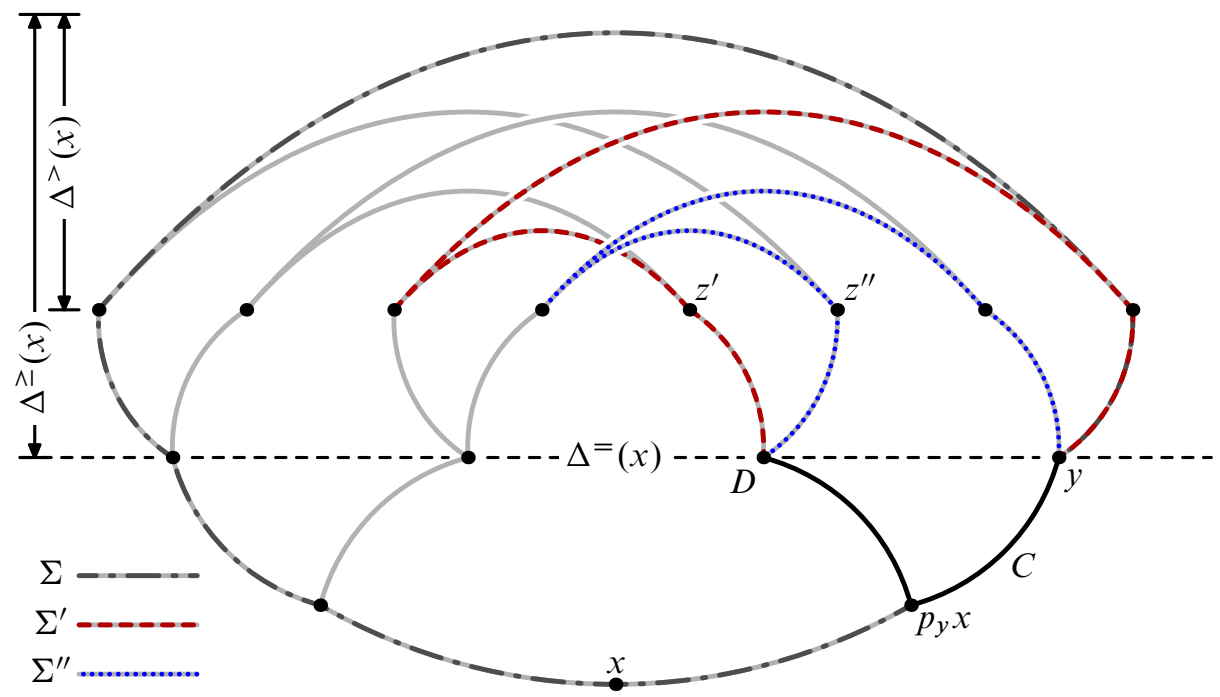

Figure 7: The construction of antipodes in $\Delta^{>}(x)$

Let $\Sigma$ be an apartment containing $x$ and $y$. Since $\Delta$ is thick, we obtain an apartment $\Sigma^{\prime}$ by Lemma 6.4 , such that $\Sigma \cap \Sigma^{\prime}=\overline{\operatorname{St}_{\Sigma} y}$. Denote by $z^{\prime}$ the vertex of $\Sigma^{\prime}$ that is opposite to $y$. According to Lemma $6.5, z^{\prime}$ is contained in $\Delta^{>}(x)$ since the intersection of $\Delta^{=}(x)$ with $\partial \mathrm{St}_{F_{1}} y$ is empty and $\operatorname{proj}_{\varnothing} z^{\prime}=z^{\prime}$. Let $C$ be a chamber of St $y$ that contains $p_{y} x$ in its closure. Denote the panel $\operatorname{proj}_{z^{\prime}} C \backslash\left\{z^{\prime}\right\}$ by $D$. By Lemma 6.4 there is an apartment $\Sigma^{\prime \prime}$ such that $\Sigma^{\prime} \cap \Sigma^{\prime \prime}=\operatorname{Conv}(C, D)$. Denote by $z^{\prime \prime}$ the vertex of $\Sigma^{\prime \prime}$ that is opposite to $y$. Then $z^{\prime \prime} \neq z^{\prime}$, since the vertices of $\operatorname{Conv}(C, D)$ are not opposite to $y$. We show that $z^{\prime \prime} \in \Delta^{>}(x)$.

By the triangle inequality we have $d\left(x, z^{\prime \prime}\right) \geq \pi / 2$. Suppose $d\left(x, z^{\prime \prime}\right)=\pi / 2$. There is a geodesic segment joining $y$ and $z^{\prime \prime}$ going through $x$ by Proposition 1.4. Then $x$ is a point of $\left[y, p_{z^{\prime \prime}} x\right]$, because $x$ can not be contained in St $z^{\prime \prime}$ according to Lemma 1.13. Since $p_{y} x$ is a point of this segment, we get $p_{z^{\prime \prime}} x=p_{z^{\prime \prime}} p_{y} x$. Observe, that $p_{z^{\prime}} p_{y} x$ is a point of $\operatorname{Conv}\left(C, \operatorname{proj}_{z^{\prime}} C\right) \cap \partial \operatorname{St} z^{\prime}=\bar{D} \in \mathcal{S}\left(\Sigma^{\prime \prime}\right)$. The retraction $\rho_{\Sigma^{\prime \prime}, C}$ on $\Sigma^{\prime \prime}$ centered at $C$ maps $p_{z^{\prime}} p_{y} x$ to $p_{z^{\prime \prime}} p_{y} x$, hence $p_{z^{\prime}} p_{y} x=p_{z^{\prime \prime}} p_{y} x$. Therefore $x$ lies on $\left[y, p_{z^{\prime}} p_{y} x\right] \subseteq \Sigma^{\prime}$. This implies $d\left(x, z^{\prime}\right)=\pi-d(x, y)=\pi / 2$ by Proposition 1.4 in contradiction to $z^{\prime} \in \Delta^{>}(x)$. 


\section{References}

[1] H Abels, Finiteness properties of certain arithmetic groups in the function field case, Israel J. Math. 76 (1991) 113-128 MR1177335

[2] H Abels, P Abramenko, On the homotopy type of subcomplexes of Tits buildings, Adv. Math. 101 (1993) 78-86 MR1239453

[3] P Abramenko, Endlichkeitseigenschaften der Gruppen $\mathrm{SL}_{n}\left(\mathbb{F}_{q}[t]\right), \mathrm{PhD}$ thesis, Frankfurt am Main (1987)

[4] P Abramenko, Twin buildings and applications to $S$-arithmetic groups, Lecture Notes in Mathematics 1641, Springer, Berlin (1996) MR1624276

[5] P Abramenko, KS Brown, Buildings, Graduate Texts in Mathematics 248, Springer, New York (2008) MR2439729

[6] P Abramenko, H Van Maldeghem, Intersections of apartments, J. Combin. Theory Ser. A 117 (2010) 440-453 MR2592893

[7] H Behr, Arithmetic groups over function fields I: A complete characterization of finitely generated and finitely presented arithmetic subgroups of reductive algebraic groups, J. Reine Angew. Math. 495 (1998) 79-118 MR1603845

[8] A Björner, Some combinatorial and algebraic properties of Coxeter complexes and Tits buildings, Adv. in Math. 52 (1984) 173-212 MR744856

[9] A Björner, Topological methods, from: "Handbook of combinatorics, Volume II", (R L Graham, M Grötschel, L Lovász, editors), Elsevier, Amsterdam (1995) 1819-1872 MR1373690

[10] M R Bridson, A Haefliger, Metric spaces of non-positive curvature, Grundl. Math. Wissen. 319, Springer, Berlin (1999) MR1744486

[11] K S Brown, Finiteness properties of groups, from: "Proceedings of the Northwestern conference on cohomology of groups (Evanston, Ill., 1985)”, volume 44 (1987) 45-75 MR885095

[12] F Buekenhout, The basic diagram of a geometry, from: "Geometries and groups (Berlin, 1981)”, (M Aigner, D Jungnickel, editors), Lecture Notes in Math. 893, Springer, Berlin (1981) 1-29 MR655057

[13] K-U Bux, K Wortman, Finiteness properties of arithmetic groups over function fields, Invent. Math. 167 (2007) 355-378 MR2270455

[14] K-U Bux, K Wortman, Connectivity properties of horospheres in Euclidean buildings and applications to finiteness properties of discrete groups, Invent. Math. 185 (2011) 395-419 MR2819164

[15] K-U Buz, R Gramlich, S Witzel, Finiteness properties of Chevalley groups over a polynomial ring over a finite field arXiv:0908.4531v4 
[16] K-U Buz, R Köhl, S Witzel, Higher finiteness properties of reductive arithmetic groups in positive characteristic: the rank theorem, Ann. of Math. 177 (2013) 311-366

[17] R Charney, A Lytchak, Metric characterizations of spherical and Euclidean buildings, Geom. Topol. 5 (2001) 521-550 MR1833752

[18] J Dymara, D Osajda, Boundaries of right-angled hyperbolic buildings, Fund. Math. 197 (2007) 123-165 MR2365885

[19] A von Heydebreck, Homotopy properties of certain complexes associated to spherical buildings, Israel J. Math. 133 (2003) 369-379 MR1968436

[20] P J Hilton, S Wylie, Homology theory: An introduction to algebraic topology, Cambridge Univ. Press, New York (1960) MR0115161

[21] B Kleiner, B Leeb, Rigidity of quasi-isometries for symmetric spaces and Euclidean buildings, Inst. Hautes Études Sci. Publ. Math. (1997) 115-197 MR1608566

[22] D Quillen, Homotopy properties of the poset of nontrivial p-subgroups of a group, Adv. in Math. 28 (1978) 101-128 MR493916

[23] B Schulz, Sphärische Unterkomplexe sphärischer Gebäude, $\mathrm{PhD}$ thesis, Frankfurt am Main (2005)

[24] EH Spanier, Algebraic topology, McGraw-Hill Book Co., New York (1966) MR0210112

[25] U Stuhler, Homological properties of certain arithmetic groups in the function field case, Invent. Math. 57 (1980) 263-281 MR568936

[26] J Tits, Buildings of spherical type and finite BN-pairs, Lecture Notes in Mathematics 386, Springer, Berlin (1974) MR0470099

[27] J Tits, Ensembles ordonnés, immeubles et sommes amalgamées, Bull. Soc. Math. Belg. Sér. A 38 (1986) 367-387 MR885545

[28] K Vogtmann, Spherical posets and homology stability for $\mathrm{O}_{n, n}$, Topology 20 (1981) 119-132 MR605652

[29] S Witzel, Finiteness properties of Chevalley groups over the Laurent polynomial ring over a finite field arXiv:1007.5216

Tulpenhofstraße 31, 63067 Offenbach, Germany

bernd.schulz@ivir.de

Proposed: Martin R Bridson

Seconded: Walter Neumann, Jean-Pierre Otal

Received: 22 August 2010

Accepted: 12 June 2012 